\title{
Analysis of correlations between polarimetric and photometric characteristics of young stars ${ }^{\star}$
}

\section{A new approach to the problem after eleven years' study}

\author{
R.V. Yudin \\ Central Astronomical Observatory of the Russian Academy of Sciences at Pulkovo, 196140 Saint-Petersburg, Russia \\ e-mail: ruslan@pulkovo.spb.su
}

Received March 12, 1999; accepted March 14, 2000

\begin{abstract}
We present the results of the investigation of correlations between the polarimetric and photometric characteristics of a sample (496 objects) of young Herbig $\mathrm{Ae} / \mathrm{Be}$ (HAEBE) stars and T Tauri (TT) stars. It is shown that, for $85 \%$ of the sample stars there is a general relation between the degree of optical polarization and the infrared colour index $(V-L)_{\text {obs }}$ and the colour excess $E(V-L)$ due to the contribution of a circumstellar dust shell. Polarimetric data were also compared with the value of $v \sin i$ to search for a possible correlation between the polarization and an inclination of circumstellar disks. Polarimetric data as well as IR excesses are considered and compared for different subgroups of young stars namely: HAEBE and TT stars with Algol-like minima of brightness (26 objects), Vega-type stars and post HAEBE stars (114 objects) and young solar-type stars (58 objects). For statistical purposes the data for young stars were compared with those collected for different groups of evolved objects such as: classical Be stars ( $\approx 300$ objects), Mira Ceti stars (39 objects), early-type supergiants from the Serkowski et al. (1975) catalogue (120 objects) and main sequence (MS) stars within $50 \mathrm{pc}$ from the Sun from the Leroy (1993) catalogue (68 objects). The value of polarization is discussed in context with the stages of evolution of circumstellar shells which were established by comparison of spectral energy distribution in the far IR (using the IRAS data). It is shown that most young stars have statistically larger value of polarization in comparison with the stars which are on a stage of evolution close to MS. We are able to contend that the changes in polarimetric behaviour of young stars are connected with evolution of their circumstellar shells.
\end{abstract}

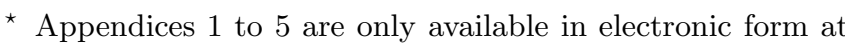
http://www.edpsciences.org
}

Key words: polarization - circumstellar matter - stars: evolution - star: pre-mains sequence

\section{Introduction}

It is well established that most of the pre-main sequence (pre-MS) stars in the range of spectral classes from early $\mathrm{B}$ to earlier F (so-called young Herbig Ae/Be stars; hereafter HAEBE) and from later $\mathrm{F}$ to $\mathrm{M}$ (so-called young $\mathrm{T}$ Tauri type stars; hereafter TT) show the variability in their polarization as well as the variability of their photometric and spectroscopic characteristics. As has been established by many observations, the intrinsic polarization of radiation is a common behaviour for most young stars (see for example Menard \& Bastien 1992; Yudin \& Evans 1998 and references therein). It is not surprising because there is now a consensus that young stars exhibit dust and gas circumstellar (CS) shells whose shape may range from spherically symmetric envelopes, through geometrically and optically thick disks, to geometrically and optically thin (narrow) disks. Polarimetry which is more sensitive to the geometry and physical conditions of the material in the vicinity of the stars may help to solve many challenging questions in a theory of circumstellar evolution. However to gain a better understanding of the processes which may take place in CS shells a statistical analysis of correlations between the polarimetric and photometric characteristics should be made. Note that to make a detailed explanation for polarimetric variability and correlations between $p$ and other characteristics of individual stars some specific processes in CS shells are often discussed (see for example Beskrovnaya et al. 1998 - presence of azimuthal inhomogeneities forming in different CS regions of a gaseous envelope; or Grinin et al. 1996; Yudin et al. 1999 - fragmentation of large comet-like 
bodies in the vicinity of a star with ejection of small dust particles). However, as a rule, a nonvariable (on a shorttime scale) component of polarization due to the presence of nonspherical dust envelopes exists in most young objects. Thus, to create a self consistent "star+shell" model for an individual object and for conclusions on the global changes of the dust distribution in CS shells of young stars the investigation of correlations between different kinds of observational characteristics (for representative groups of the objects which are on the different or similar stages of evolution) is most important. Such investigations have much potential for yielding information on classification criteria. As a result this may lead to a significant change in our knowledge of star and "star+shell" evolution.

The general aim of the proposed work is to compare all available polarimetric data for young stars with their photometric behaviour in the near and far IR, thus establishing real correlations between polarization and stages of their circumstellar shells evolution. Justification for this work is that since 1985-1988 numerous data on the polarimetry of different type young stars have been obtained. On the other hand, the lists of TT and HAEBE stars were re-established and many new objects were re-classified as young stars (Thé et al. 1994). In addition, new hypotheses on CS shells' evolution have been considered in the past (mainly in connection with changes in the SED) (see for example Malfait et al. 1998). In Sect. 2 we discuss briefly the results of previous studies of correlations between polarization and other observational characteristics of young stars. The accumulated lists of HAEBE and TT stars with available polarimetric and photometric data are presented in the Appendices 1-5. In Sect. 3, new correlation dependencies between $\log p$ and IR colour excesses are investigated for different groups of young stellar objects (HAEBE and TT stars, young solar-type and Vega-type stars, HAEBE stars with Algol-like minima of brightness). The histograms of polarization and near IR excesses distributions for the above mentioned groups are compared. In Sect. 4 the polarization for different type stars is discussed in context with their projected rotational velocities. A comparison of this behaviour between the young stars and more evolved classical Be stars is made. For different types of stars which exhibit polarization of radiation and near IR excesses but are on a different stage of evolution (classical Be stars, red giants and supergiants, supergiants of early spectral types, peculiar B[e] stars, MS stars in solar neighborhood) their location at the $\log p / E(V-L)$ diagram are investigated in Sect. 5 and compared with the behaviour in young stars. In Sect. 6 main mechanisms of polarization in young stars are discussed, also simple calculations of expected polarization in the framework of a dust model are made. Some astrophysical aspects of the $\log p / E(V-L)$ diagram constructed here like that of evolutionary changes in CS shells of low- and intermediate mass young stars are discussed in Sect. 7 . In Sect. 8 po- larization of young stars is investigated in the context of their spectral energy distributions in the near and far IR.

\section{Previous studies of correlations between polarimetric and photometric data for young stars}

The first attempt to compare the observed polarimetric characteristics of young TT stars with the colour indices $V-R, V-I, V-J, V-H, V-K, V-L, V-M$ and $V-N$ (Bastien 1982) led to detection of a correlation between $p$ and the IR colour indices. In a following paper (Bastien 1985) a statistical analysis of similar data on the polarization of 85 TT stars was made and correlations between $p$ and the IR colour indices and the colour excess $[V-L]$ was found. Note however that the correlation coefficients obtained by Bastien (1985) were small and not significant in some cases. Nevertheless the main conclusion of Bastien (1985) was as follows:

The polarization is well correlated with IR colour indices and colour excesses, implying that most of the IR excesses are due to the same dust grains which are responsible for the polarization.

A little later, the comprehensive analysis of correlation between $p$ and photometric characteristics of young stars of different types (TT and HAEBE stars) was fulfilled by Yudin (1988). In the framework of his study a correlation between $p$ and IR colour excesses $E(V-J), E(V-H)$, $E(V-K), E(V-L), E(V-M)$ was investigated (where these colour excesses were calculated using the following formula:

$E(V-L)=(V-L)_{\mathrm{obs}}-(V-L)_{0}-\left(A_{V}-A_{L}\right)$

and $(V-L)_{0}$ is the normal colour index corresponding to the spectral type of the star, $A_{V}$ and $A_{L}$ are the amount of interstellar absorption in the respective photometric bands). It is important that $E(V-L)$ determines the relative contribution of the circumstellar shell to the IR emission in comparison with the stellar photosphere in the given IR band.

As a result of the work of Yudin (1988) two main conclusions were made:

1. Common linear dependencies of $\log p$ on the IR colour excesses are exhibited for $80 \%$ of young stars in both types (TT and HAEBE), and the correlation coefficients of these dependencies were high (up to 0.93);

2. Dust with a temperature of $\approx 900 \mathrm{~K}$ (i.e. dust which is responsible for IR excesses in $L$ and $M$ bands) makes the maximum contribution to the optical polarization of young stars.

Moreover, it was also noted that the $\log p / E(V-L)$ diagram can provide a criterion for selecting stars that are undergoing pre-MS evolution. This conclusion was made on the basis of a comparison between the position of young stars as well as Be stars and red giants on the above mentioned diagram. 
Another important point is that in the first paper Bastien (1982) advanced the suggestion that stars with more highly polarized radiation are younger, but later (Bastien 1985) he rejected his own suggestion. Yudin (1988) however showed that more young stars have more highly polarized emission, on average, and suggested that probably polarization of young stars originating in the circumstellar dust shells decreases as the stars evolved toward the MS.

At approximately the same time Tamura \& Sato (1989) discussed the changes in IR polarization of TT stars in terms of evolutionary changes in the optical thickness of their circumstellar disks. They showed that "the IR polarization is caused by CS dust grains around TT stars and there is a positive correlation between the IR polarization and colour excess while no correlation is found between polarization and reddening". They also suggested a possible evolutionary sequence in the IR polarization of young stellar objects.

Later Yudin (1990) showed that there is a certain sequence in the CS shell evolution of different types of young stars in the IRAS colour-colour diagram and tried to discuss the changes in optical polarization of young stars in this context. This approach was also recently applied to the analysis of the evolutionary status for the large group of early-type young stars and candidate members by Yudin \& Evans (1998).

The bank of polarimetric data (available at present) for young stars allows us to make a new statistically significant investigation of evolutionary changes in polarization, taking into account new results in the theory of CS shell evolution.

\section{Polarization and near IR excess}

As was mentioned above the investigation of $p$ and IR colour indices was fulfilled by Bastien (1985) for $85 \mathrm{TT}$ stars, however the correlation coefficients for " $p$-colour indices" dependencies were calculated by him only for about 50 of the stars from his sample. Further, Yudin (1988) studied similar correlations for a sample of about $70 \mathrm{TT}$ and HAEBE stars and calculated the correlation coefficients for his dependencies for about 50 stars too. In spite of the fact that Yudin (1988) has studied not only TT but also HAEBE stars the same number of used stars was justified due to a limited (at that time) data on interstellar absorption and IR photometry. Here we should try to construct a similar $\log p / E(V-L)$ diagram adding all new available data which have been collected since 1988 .

\subsection{Sample of stars}

All collected data are presented in the Appendices 1-5 for HAEBE stars: [1], Vega-type and post HAEBE stars: [2],
HAEBE and TT stars with synchronous photometric and polarimetric measurements: [3], TT stars: [4], and young solar-type stars: [5]. The columns in these Tables give the star's name, the weighted average value of $p$ (preferable in $V, R$ bands for all published data) in percent, the full amplitude of polarimetric variability and the references, the spectral class of a star and the references, the average value of the observed colour index $(V-L)_{\text {obs }}$ in magnitudes and the references, the average value of $v \sin i$ in $\mathrm{km} \mathrm{s}^{-1}$ and the references, the calculated value of colour excess $E(V-L)$ in magnitudes and remarks. As may be noted from the Appendices 1-5 the number of HAEBE stars (including candidate members and Vegaand $\beta$ Pic type stars) and TT stars (including young solartype stars) which are used in this study is 264 and 232 respectively. The number of stars with all data which are needed for statistical analysis (i.e. $(V-L)_{\text {obs }}$, Sp classes, $p$ and $\left.A_{V}\right)$ is about 400, eight times greater than in previous studies.

\subsection{The new $\log p / E(V-L)$ diagram}

First of all we plotted, in Fig. 1, the observed polarization for the sample of selected young stars against their observed $(V-L)_{\text {obs }}$ colour index. We emphasize that pronounced correlation between $\log p$ and $(V-L)_{\text {obs }}$ for a sample of 402 young stars is evident. On the other hand Fig. 1 demonstrates no noticeable differences in position of different types of young objects (HAEBE and TT stars) on the diagram. It is easy to explain while the values $(V-L)_{\text {obs }}$ are composed of normal colour indices corresponding to the spectral type of stars and biased by interstellar absorption. However, it follows even from this relation that most of the Vega-type and young solar-type stars are well concentrated in the left side of the diagram and represent a separate group.

In the next step we construct the diagram $\log p / E(V-$ $L)$ separately for HAEBE stars (Fig. 2) and for TT stars (Fig. 3), where $E(V-L)$ was calculated in the same way as mentioned in Sect. 2 (i.e. corrected for the normal colour index corresponding to the spectral type of the star and the amount of interstellar absorption, see (1)).

From Fig. 2 we conclude that most HAEBE stars (as many as $85 \%$ ) show a clear dependence between the polarization degree and near IR excess. The behaviour for TT stars is not so apparent. However some tendency to increase $p$ with increasing near IR excess exists, (see Fig. 3) and more than $80 \%$ of TT stars are concentrated at the same region of the diagram as HAEBE stars.

In Fig. 4 all data for young stars from our sample are plotted together.

It is clear that most young stars are well concentrated along a line with the pronounced positive correlation between $\log p$ and $E(V-L)$.

As was suggested by Herbig (1960), the so-called HAEBE 


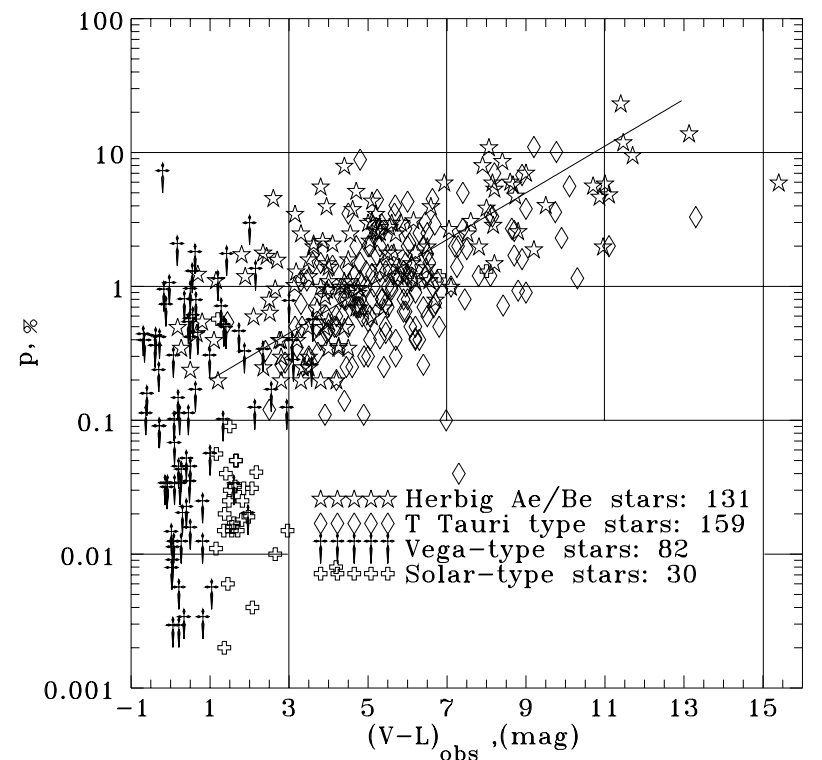

Fig. 1. $\log p /(V-L)_{\text {obs }}$ diagram for HAEBE, TT type, Vegatype and young solar-type stars. The number of stars used is indicated. The solid line represents a linear fit for the region $1^{\mathrm{m}}<(V-L)_{\mathrm{obs}}<15^{\mathrm{m}}$

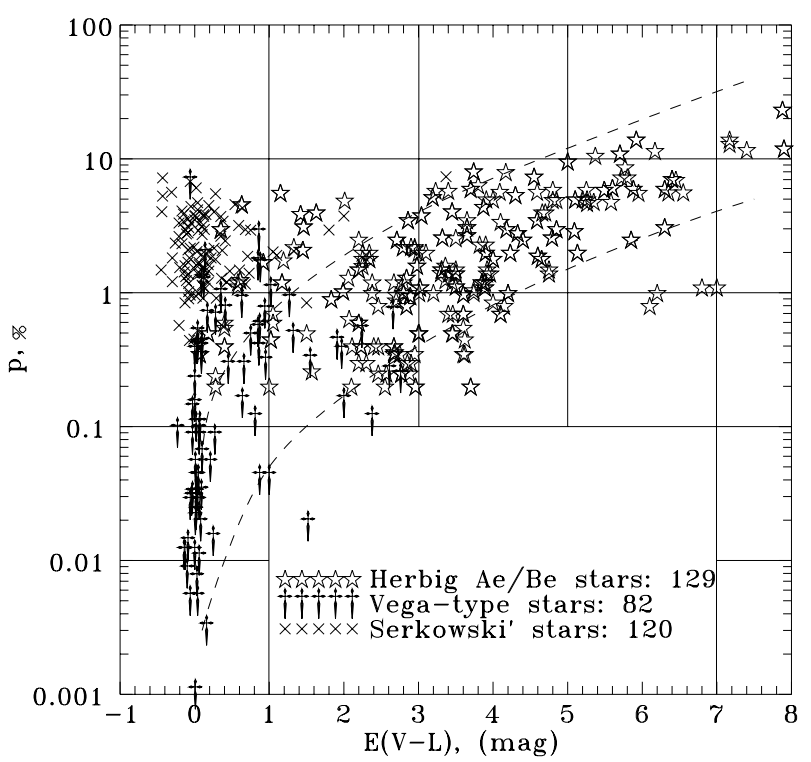

Fig. 2. $\log p / E(V-L)$ diagram for HAEBE, Vega-type stars and standard early-type stars from Serkowski et al. (1975). The dashed lines indicate the bounded region of the diagram in which $85 \%$ of HAEBE stars are located

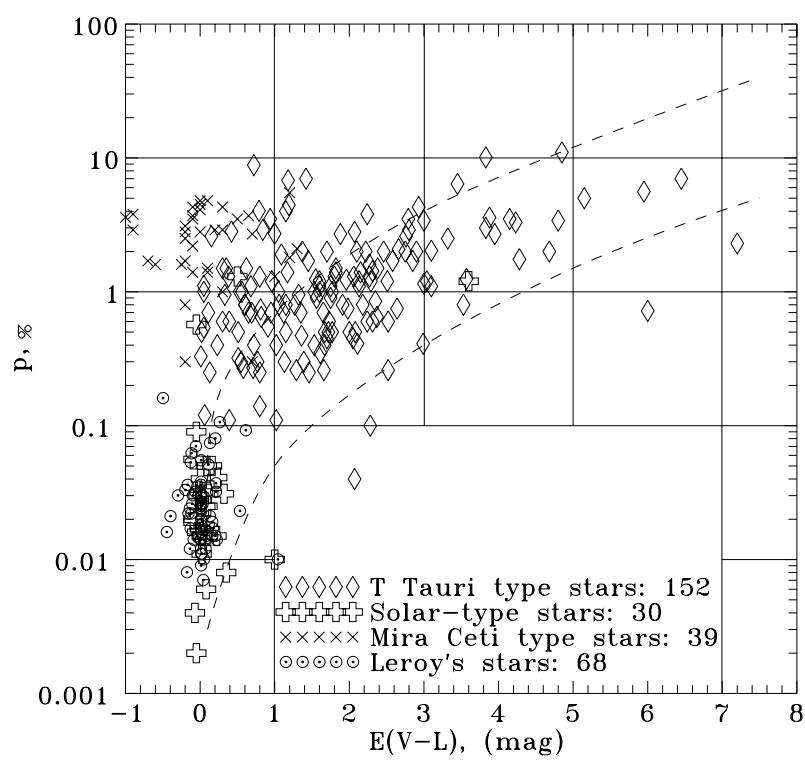

Fig. 3. $\log p / E(V-L)$ diagram for TT stars, young solar-type stars and Mira Ceti-type stars and stars within $50 \mathrm{pc}$ from the Sun from Leroy (1993). The dashed lines indicate the bounded region of the diagram in which $80 \%$ of $\mathrm{TT}$ stars are located

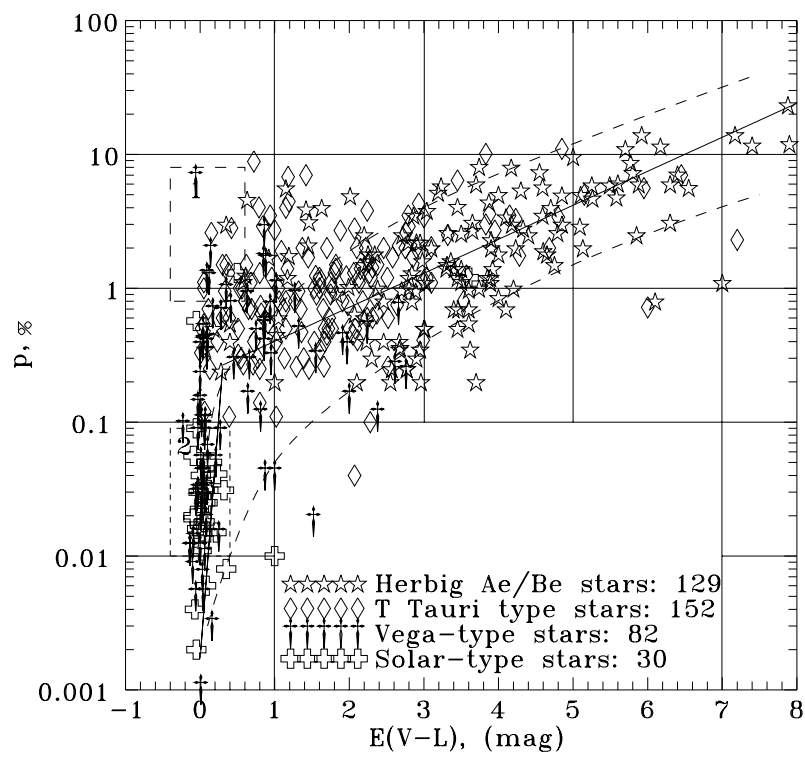

Fig. 4. The common $\log p / E(V-L)$ diagram for HAEBE, TT, Vega-type stars and young solar-type stars. The boxes 1 and 2 indicate the position of supergiants from Serkowski et al. (1975) and MS stars from Leroy (1993) respectively. The dashed lines indicate the bounded region of the diagram in which $85 \%$ of HAEBE and TT stars are located. The solid line represents a linear fit for the dependence: Eq. (3) 
stars are intermediate mass analogues of young TT stars in the mass range between 2 and $8 M_{\odot}$. From that time numerous observational and theoretical confirmations of this suggestion have been discussed and there is no doubt at present that the CS environment of TT and HAEBE stars is rather similar (in the sense of non-spherical symmetry). Note however that some significant differences in physical conditions in CS shells exist. For example Grinin (1998) noted that "HAEBE stars are more massive objects than TT stars and their CS disks are geometrically thicker". Besides he also noted that for TT stars the model of magnetospheric accretion is successfully adopted whereas their application for HAEBE stars faced several problems. Moreover, Pogodin (1998) have pointed out several main distinctions between the disks around classical TT stars and HAEBE stars as follows:

1. The inner boundary of the HAEBE star's disk is not as close to the star as in the case of TT stars;

2. The gas density is lower in the case of the HAEBE disk; and

3. Matter infall in HAEBE stars is discrete rather than continuous.

In addition note that although we know that TT stars are surrounded by CS accretion disks, there is still active debate going as to whether or not HAEBE stars have accretion disks (see for example Böhm \& Catala 1994).

The $\log p / E(V-L)$ diagram discussed here provides additional information on similarities and differences in CS shells in both kinds of young objects because the polarization and near IR excesses both originate mainly in the inner part of CS shells and are sensitive in general to the presence of hot dust in the CS environment.

The main conclusions drawn from the diagram constructed here (see Figs. 2-4) are as follows:

First, most of HAEBE and TT stars show a common linear dependence between the $\log p$ and near IR excess. In the range of $E(V-L)$ from $1^{\mathrm{m}}$ to $8^{\mathrm{m}}$ the best fit for this dependence (solid line in Fig. 4) may be depicted by the following equation:

$p \approx 0.22 \mathrm{e}^{0.58 E(V-L)}$

or

$\log p \approx 0.25 E(V-L)-0.66$.

This is in good agreement with earlier results of Yudin (1988) (taking into account the much lower statistics in the previous study). In the range $E(V-L)<1^{\mathrm{m}}$ the relation is quite different. The behaviour for this region is more complicated and probably may be depicted by the equation in the form:

$p \approx k \times E(V-L)$.

More detailed analysis of this behaviour will be considered in Sect. 6.

Second, on average HAEBE stars (131 objects) exhibit larger near IR excesses than TT stars (153 objects) (see Figs. 5-6). For HAEBE stars the mean value of
Table 1. Numerical differences between polarization distributions for HAEBE and TT stars

\begin{tabular}{|c|c|c|c|c|c|}
\hline & \multicolumn{5}{|c|}{$p<1.5 \% p>1 \% p>2 \% p>3 \% p>4 \%$} \\
\hline $\mathrm{TT}$ & & & 23.8 & $11.7 \%$ & $6.5^{\mathrm{c}}$ \\
\hline HAEBE & $49.6 \%$ & $64.5 \%$ & $41.0 \%$ & $27.6 \%$ & $19.2 \%$ \\
\hline
\end{tabular}

$E(V-L)$ is about $\approx 3^{\mathrm{m}} .48$ (with the standard deviation $\left.\sigma_{E(V-L)} \approx 1^{\mathrm{m}} .67\right)$ and $76 \%$ of stars exhibit $E(V-L)$ excesses ranging between $2^{\mathrm{m}}$ and $6^{\mathrm{m}}$. For TT stars the mean value of $E(V-L)$ is about $\approx 1^{\mathrm{m}} .85\left(\sigma_{E(V-L)} \approx 1^{\mathrm{m}} .33\right)$ and $90 \%$ of TT stars exhibit $0^{\mathrm{m}}<E(V-L)<3^{\mathrm{m}}$.

Third, clear differences are found also in the polarization distribution for the groups of TT (174 objects without young solar-type stars) and HAEBE stars (149 objects without Vega-type stars) in common (see Figs. 7-8). Note, that the polarization distribution has been investigated for 122 TT stars and related objects by Menard \& Bastien (1992) (it is peaked at $0.75 \%$ with the average polarization of $1.7 \%$ ) and the distribution constructed here for $174 \mathrm{TT}$ stars is very similar. For TT stars the mean value of polarization is about $1.6 \%$ (with the standard deviation $1.8 \%$ ) and for HAEBE stars the mean value of $p$ is $\approx 3.0 \%\left(\sigma_{p} \approx 3.4 \%\right)$. For both TT and HAEBE stars the polarization distributions are broad and there are tails in the distributions, as is evident from the fact that the standard deviation in both cases is greater than the mean. The differences are much pronounced in numerical form (see Table 1$)$. It is easy seen that a significant fraction $(1 / 3)$ of HAEBE stars exhibit polarization degree at a level higher than $3 \%$ whereas among TT stars only $1 / 8$ show the same level of polarization.

Fourth, most of the young early-type stars which might be classified as Vega-type, $\beta$-Pic type or post HAEBE stars (i.e. comparable young stars near the end of the pre-MS evolutionary phase or young MS stars) show statistically smaller near IR excesses and polarization than HAEBE stars (see Fig. 2). The same conclusion can be made for young solar-type stars (see Fig. 3). The histograms of near-IR excess and polarization distributions for Vega-type and young solar-type stars are presented in Figs. 9 and 10. As follows from Fig. 9 about $65 \%$ of Vega-type and solar-type stars exhibit near-IR excess less than $0^{\mathrm{m}} .25,72 \%$ - less than 0.5 and $85 \%$ - less than $1^{\mathrm{m}}$. In the polarization distribution about $63 \%$ of Vega-type and solar-type stars show polarization degree less than $0.1 \%, 71 \%$ - less than $0.25 \%$ and $90 \%$ of them have $p<0.75 \%$. Note that a portion of post HAEBE and Vega-type stars in our sample are stars in the Orion region and they exhibit relatively large observed polarization at the level of $p_{\text {obs }} \approx 0.4-1 \%$ that is not consistent with their small near IR excesses (see Appendix 2). However, the analysis of interstellar polarization in the Orion region yields $p_{\text {is }} \approx 0.25 \%$ for the majority of cluster stars 


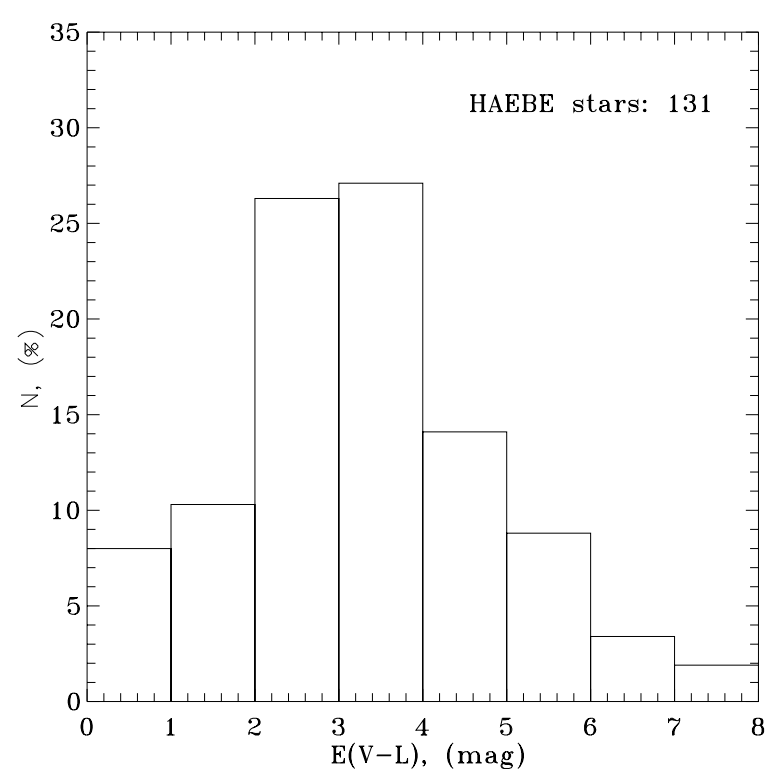

Fig. 5. Histogram of near IR excesses for HAEBE stars

(Breger et al. 1981). Taking into account this value, the intrinsic polarization for most of these Orion stars would not exceed $0.2 \%$. This leads to the a significant improvement in the correlation in the sense that these stars would transit to the lower left region of the diagram. Besides, in the polarization distribution the number of post HAEBE and Vega-type stars with the polarization degree less than $0.25 \%$ would reach a value of about $80 \%$.

One can note however that the spread of the data points for young stars around the average is not small. There are a few reasons to account for such behaviour:

1. In spite of significant photometric variability taking place in many young stars the average value of $(V-L)_{\text {obs }}$ indices may be well determined. Some spread in estimates of Sp types and interstellar extinction taken from the literature is more significant. However for our statistical investigation this is not so important since as a rule in the modelling of the SED the use of an earlier Sp type requires one the large value of reddening. Few typical examples for the stars with strong discrepancy in determination of $\mathrm{Sp}$ type and $A_{V}$ are given in Table 2 . As can be seen in Table 2 no strong variations in $E(V-L)$ occurred due to different estimates of Sp types and $A_{V}$. Note however that for some objects (especially for TT stars) uncertainties in estimates of Sp classes and $A_{V}$ may lead to the significant scattering of the data points;

2. More important is that, in contrast to photometric observations, the polarimetric data are very limited. The values of $\mathrm{p}$ which are presented in the Appendices are often an average from 2-3 measurements or even a single one. However, as has been pointed out by Menard \& Bastien

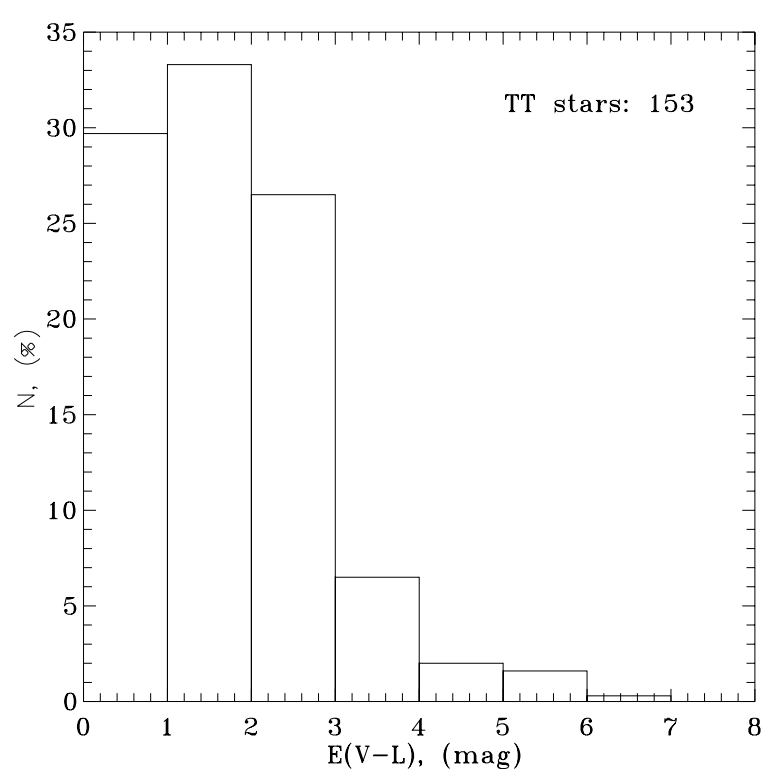

Fig. 6. Histogram of near IR excesses for TT type stars

(1992) and Yudin \& Evans (1998), about $100 \%$ of all young stars are polarimetrically variable. This may lead to the displacement of their location on the diagram;

3. In some cases averaging of polarization over a long period is not correct since a star may usually possess small polarization and show an increase of $p$ from time to time on a short time scale. Such behaviour is observed in HAEBE stars with Algol-like minima. Their location on the diagram will be considered in the next section;

4 . For most of the stars we use the observed values of polarization without allowance for interstellar polarization;

5 . Finally the scattering of the data points on the diagram may be due to a specific orientation of nonspherical dust shells surrounding some stars. This possibility will be considered in Sect. 4.

To investigate the influence of interstellar polarization on our resulting dependence we try to calculate an interstellar polarization component for a few stars which lie far from the average line and/or on the edge of the region. The values of interstellar and intrinsic components for some stars were derived by the field stars method using the VizieR Service at the CDS (using the catalogues of Mathewson et al. 1978 and Axon \& Ellis 1976) or were taken from literature and are tabulated in Table 3 . The results of these calculations were used to investigate the changes in the position of stars on the diagram. As can be seen in Fig. 11 the plotting of the values of intrinsic polarization results in the transition of a few selected stars close to the average line into the region occupied by most young stars. For TT stars which usually exhibit relatively low levels of intrinsic polarization the estimate of interstellar 


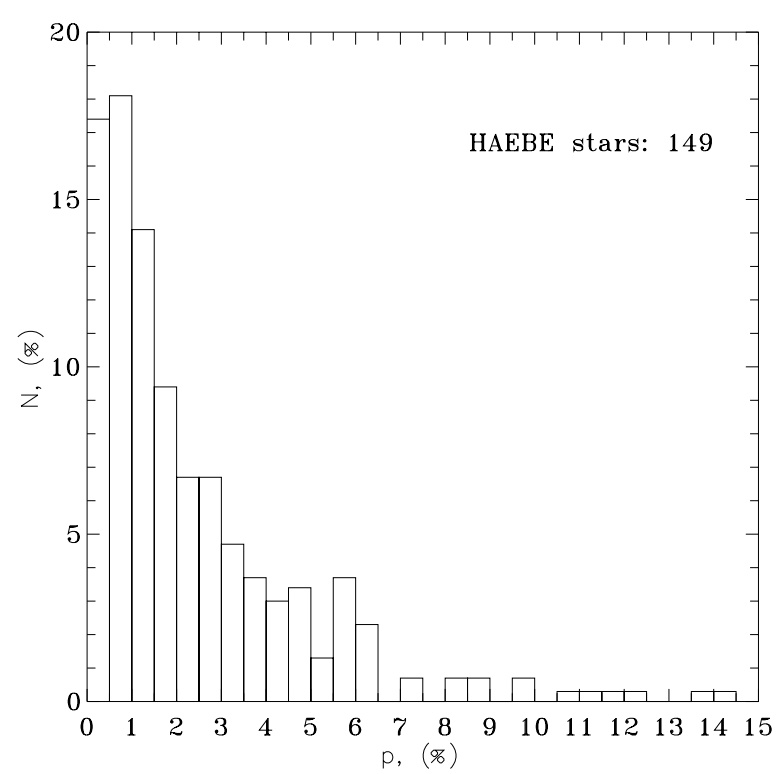

Fig. 7. Histogram of polarization for HAEBE stars

components is very important. The objects whose positions strongly deviate from others on the diagram are mainly TT stars and they usually show much higher values of polarization than might be expected from their near IR excesses (see for example: $\mathrm{LkH} \alpha 338, \mathrm{LkH} \alpha 332-20, \mathrm{Sz} 30$, Sz 45, CT Cha, VV Cha, Haro 1-1, CHX 10A, CED 110, ROX 7, ROX 39, ROX 42B, ROX 43AB, P1540, P 1724 and AZ Ori). However most of these "peculiar" stars are located in the Chamaeleon and Ophiuhus regions or in the Orion nebula and their large observed polarization may be due to significant interstellar polarization (see for example Whittet et al. 1991; Breger 1976, 1977). As follows from Whittet et al. (1994) namely for Cha I the considerable uniformity of polarization vectors in this region is observed and the large group of young stars in Cha I show unexpectedly high polarization degree in comparison with their relatively low near IR excesses. This fact has been discussed for CHX 10A by Whittet et al. (1994) who suggested that "for this weak-lined TT star the interstellar polarization component dominates over any intrinsic component". Other "peculiar" stars were measured polarimetrically on a single occasion and estimates of their polarization may be doubtful. Moreover, $\mathrm{Sz} 30$, VV Cha, CHX 10, $\mathrm{LkH} \alpha 332-20$ are well-known close binaries which may also affect on their observed polarization. Nevertheless the fraction of stars which deviate from the general dependence is not large and there is no need to invoke another mechanism of polarization instead of the scattering in nonspherical dust shells for the majority of young stars.

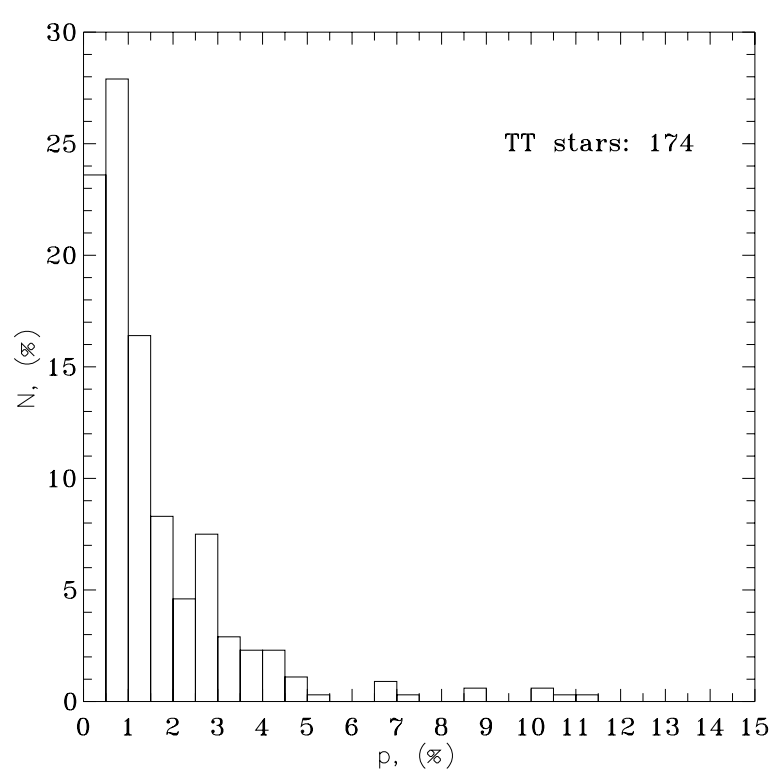

Fig. 8. Histogram of polarization for TT type stars

Table 2. The calculated values of $E(V-L)$ for different estimates of spectral types and interstellar extinction for selected stars

\begin{tabular}{ccccccc}
\hline name & Sp & ref. & $A_{V}$ & ref. & $(V-L)_{\text {obs }}$ & $E(V-L)$ \\
\hline LkH $\alpha 208$ & B7 & 1 & 1.7 & 1 & 5.5 & 4.2 \\
& F0 & 2 & 0.4 & 2 & 5.5 & 4.3 \\
V 376 Cas & F0 & 1 & 2.9 & 1 & 11.1 & 6.75 \\
& B5 & 2 & 5.2 & 2 & 11.1 & 7.55 \\
T Ori & B9 & 1 & 1.7 & 1 & 5.3 & 4.00 \\
& A3 & 3 & 1.1 & 3 & 5.3 & 3.92 \\
\hline
\end{tabular}

1 - Hillenbrand 1992; 2 - Cohen \& Kuhi 1979; 3 - Brooke et al. 1993.

\subsection{HAEBE and TT stars with Algol-like minima}

It is also interesting to investigate the behaviour on the $\log p / E(V-L)$ diagram for the stars with available data of synchronous polarimetry and photometry. Most of these objects (so-called HAEBE stars with Algol-like minima of brightness, hereafter UXOrs) were investigated by Grinin and coauthors (Grinin 1991; Grinin et al. 1991) and some objects by Yudin (1992), Yudin \& Evans (1998). One can readily see in Fig. 12 that most of them are transferred along the main dependence within the region in which $90 \%$ of all young stars are located. For each of the objects we use average values of $p$ and colour excesses for a few stages of brightness. Moreover, for BF Ori, CQ Tau, RR Tau and BM And we use the values of intrinsic polarization which were calculated using the estimates of interstellar components (see references to the objects in Appendix 3). Of course in some cases the changes in polarization during the 


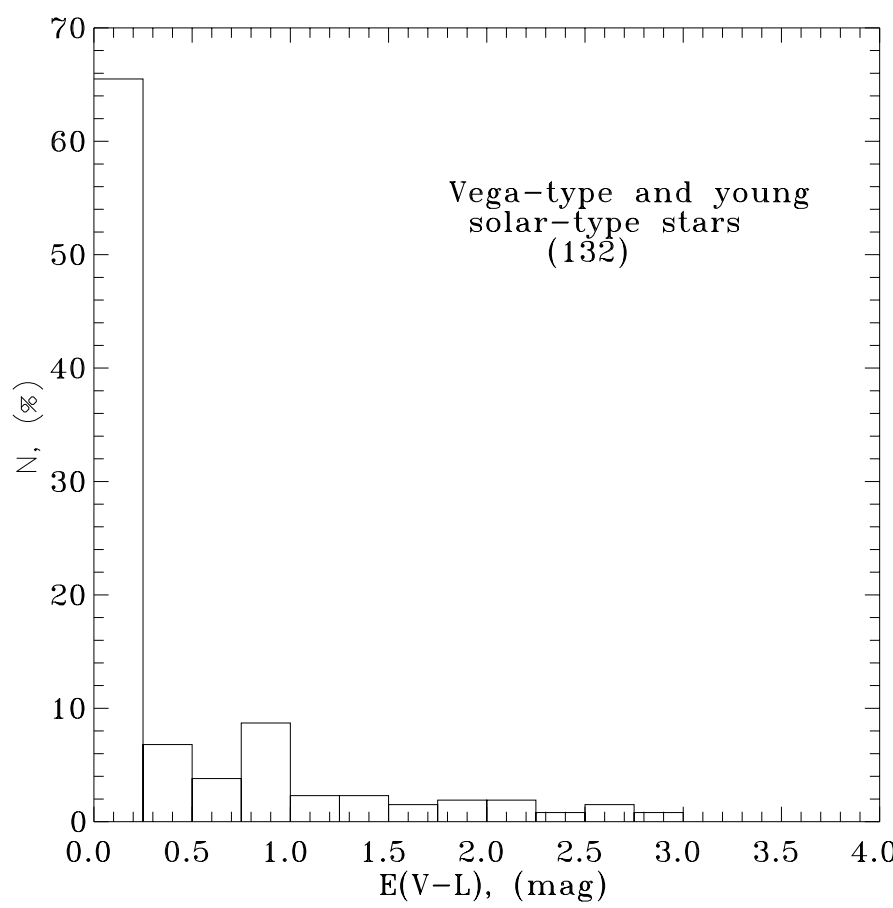

Fig. 9. Histogram of near IR excesses for Vega-type stars and young solar-type stars

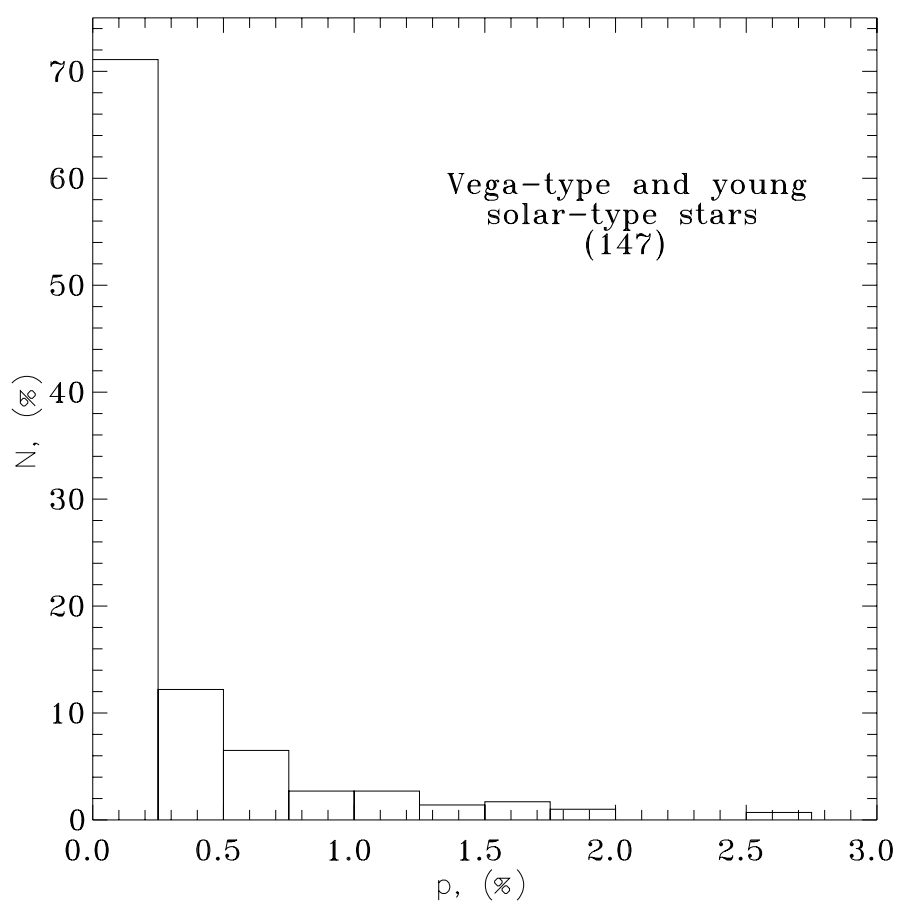

Fig. 10. Histogram of polarization for Vega-type stars and young solar-type stars
Table 3. Estimates of intrinsic polarization for some stars

\begin{tabular}{|c|c|c|c|c|}
\hline name & $p_{\mathrm{obs}}, \theta_{\mathrm{obs}}$ & $p_{\text {is }}, \theta_{\text {is }}$ & $p_{\text {int }}, \theta_{\text {int }}$ & rem. \\
\hline MWC 137 & $6.0 \% 163^{\circ}$ & $4.0 \% 162^{\circ}$ & $2.0 \% 162^{\circ}$ & \\
\hline HD 45677 & $1.0 \% 166^{\circ}$ & $0.8 \% 75^{\circ}$ & $1.7 \% 166^{\circ}$ & 1 \\
\hline HD 316285 & $2.9 \% 166^{\circ}$ & $2.5 \% 165^{\circ}$ & $0.4 \% 165^{\circ}$ & \\
\hline HD 150193 & $5.0 \% \quad 48^{\circ}$ & $2.5 \% 21^{\circ}$ & $4.2 \% \quad 65^{\circ}$ & \\
\hline HD 100546 & $0.2 \% \quad 60^{\circ}$ & $0.6 \% 117^{\circ}$ & $0.8 \% 30^{\circ}$ & 4 \\
\hline HD 259440 & $3.5 \% 165^{\circ}$ & $1.5 \% 165^{\circ}$ & $2.0 \% 165^{\circ}$ & \\
\hline HD 163296 & $0.2 \% 28^{\circ}$ & $0.6 \% 175^{\circ}$ & $0.6 \% 75^{\circ}$ & 2 \\
\hline ВНJ 71 & $4.0 \% 128^{\circ}$ & $2.5 \% 100^{\circ}$ & $3.3 \% 145^{\circ}$ & 5 \\
\hline IL Cep & $4.6 \% 115^{\circ}$ & $2.5 \% 100^{\circ}$ & $2.8 \% 128^{\circ}$ & 5 \\
\hline HD 142666 & $0.6 \% 78^{\circ}$ & & $0.5 \% \quad 70^{\circ}$ & 6 \\
\hline HD 141569 & $0.6 \% 90^{\circ}$ & & $<0.15 \% 105^{\circ}$ & 6 \\
\hline HD 53367 & $0.6 \% 44^{\circ}$ & & $0.2 \% \quad 44^{\circ}$ & 1 \\
\hline HD 37806 & $0.5 \% 120^{\circ}$ & $0.4 \% \quad 76^{\circ}$ & $0.6 \% 138^{\circ}$ & 1 \\
\hline HD 87643 & $0.9 \% 165^{\circ}$ & $2.2 \% 155^{\circ}$ & $1.3 \% 65^{\circ}$ & \\
\hline RCW 34 & $5.6 \% 59^{\circ}$ & $3.5 \% 95^{\circ}$ & $5.0 \% 75^{\circ}$ & 3 \\
\hline HD 98922 & $0.2 \% 160^{\circ}$ & $0.3 \% 120^{\circ}$ & $0.33 \% 12^{\circ}$ & \\
\hline
\end{tabular}

1 - The values of interstellar polarization from Oudmaijer \& Drew (1999); 2 - none stars closer than 500 pc around the object, the estimate of interstellar component has been made using 6 stars with $A_{V} \approx 0$ m $4 ; 3$ - the values of interstellar polarization adopted for the distance $D>1800 \mathrm{pc} ; 4$ - Clarke et al. (1999); 5 - IL Cep and BHJ 71 lie close one to another in the ski plane within 1.5. The values of interstellar polarization adopted for the distance of about $1 \mathrm{kpc} 6$ - see Yudin et al. (1999).

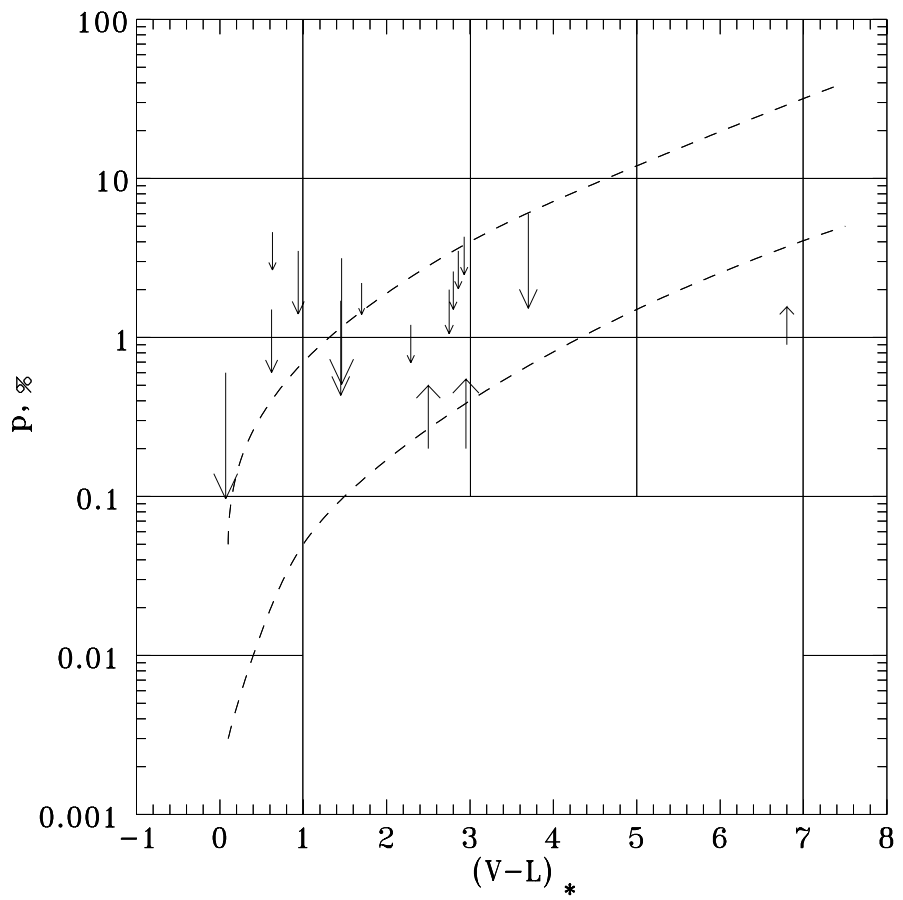

Fig. 11. Changes in the position on the $\log p / E(V-L)$ diagram of some young stars taking into account their intrinsic polarization 


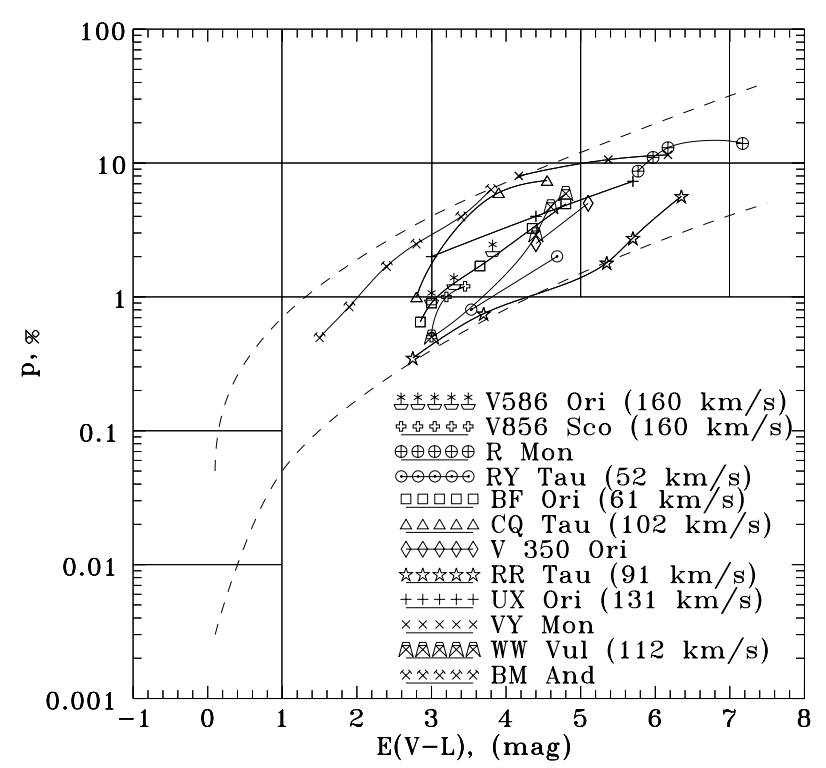

Fig. 12. $\log p / E(V-L)$ diagram for HAEBE stars with Algol-like minima

optical fading may have more complicated behaviour (see for example Grinin 1988; Grinin et al. 1991; Voshchinnikov \& Grinin 1988; Grinin et al. 1995). Nevertheless, for the most part this behaviour substantiates the validity of the dependence discussed here. The only assumption has been made here, namely that the IR fluxes of these stars are not changing strongly in comparison with the optical variations during the optical fading. Variations of IR fluxes for some HAEBE stars (including UXOrs: BF and UX Ori) were detected by Hutchinson et al. (1994). However examination of the Kilkenny et al. (1985) catalogue indicates that the amplitude of photometric variability of young stars in the optical region of spectra is in general much larger than that of variability in the IR region. The same conclusion is evident from the paper of Davies et al. (1990). Thus the above mentioned assumption would not affect strongly the behaviour of the stars with Algol-like minima on the diagram. Finally note that behaviour like that observed for UXOrs has been detected in few TT stars (see Appendix 3) and their transfer on the diagram takes place again within the same region and along the main dependence. This fact indicates again similarity of polarization mechanisms at least in some TT and HAEBE stars.

\subsection{Young solar-type and Vega-type stars}

Our sample of young stars has also included a small group of young solar-type stars (see Appendix 5). Most of them are young MS stars and no near IR excesses are observed for them. The absence of hot dust in their envelopes led to the suggestion that the main mechanism of polarization is not connected with dust and the intrinsic polarization in $U$ and $B$ bands is caused by magnetic activity of these stars. However in the red ( $R$ and $I$ bands and possibly in $V$ ) the polarization can originate in CS shells with large scattering particles (see Huovelin 1985). Since all selected stars are located within 50 pc from the Sun, the interstellar extinction and interstellar polarization are negligible. An inspection of the catalogue of Leroy (1993) immediately indicates a small level of polarization for all stars of this type $(p<0.05 \%)$ and these stars are located in the lower left side of the diagram (see Fig. 3). One can note that these stars may be later spectral type analogues of Vega-type or $\beta$ Pic type stars (see Appendix 2) which are located in the same region of the diagram (see Figs. 2 and 4). The prototypes for these stars (Vega, $\beta$ Pic and Fomalhaut) are classified as the young MS stars and they are characterized by the existence of disk-like dust envelopes. A brief discussion on polarimetric properties of some $\beta$ Pic-type stars has been presented recently by Yudin et al. (1999). In spite of the presence of cool dust in their CS disks the polarization of these stars is very small even for $\beta$ Pic itself for which the CS disk is viewed edge-on. Two possibilities to explain this behaviour exist: - an absence of a sufficient amount of hot dust (which is a primary source of polarization in most young stars) in their CS shells i.e. the disks are optically thin and the unpolarized starlight dominates; and

- small geometrical thickness of the disks around them, that is unfavorable for producing polarization (see for example Dolginov et al. 1995).

Note however, that the group of Vega-type stars is not homogeneous. Some objects might be considered as genuinely MS stars and they have small polarization $(p<0.1 \%)$ and near-IR excess $0^{\mathrm{m}}<E(V-L)<1^{\mathrm{m}}$, whereas others have $1^{\mathrm{m}}<E(V-L)<3^{\mathrm{m}}$ and a higher level of polarization $(0.1 \%<p<0.7 \%)$. The latest group includes the stars: HD 31648, HD 34282, HD 37411, HD 38120, HD 57150, 51 Oph, HD 139614, HD 142666, HD 144432, HD 169142 and others. Most of them have been studied early as the members of HAEBE group and there are no doubts on their youth. Some stars have been investigated recently as Vega-type candidates by Coulson et al. (1998) who suggested that "these stars are on an earlier evolutionary state then the archetypes". It is felt that these objects are in the transition stage from HAEBE stars to young MS stars (see further discussion in Sect. 7). The same suggestion has been made recently for HD 142666 and HD 139614 by Yudin et al. (1999). In addition note that HD 31648 and HD 163296 (these stars also show polarization at the level of about $0.2 \%$ and the value of $\left.E(V-L) \approx 3^{\mathrm{m}}\right)$ were recently considered as members of "baby" $\beta$ Pic type stars (Sitko et al. 1999). 


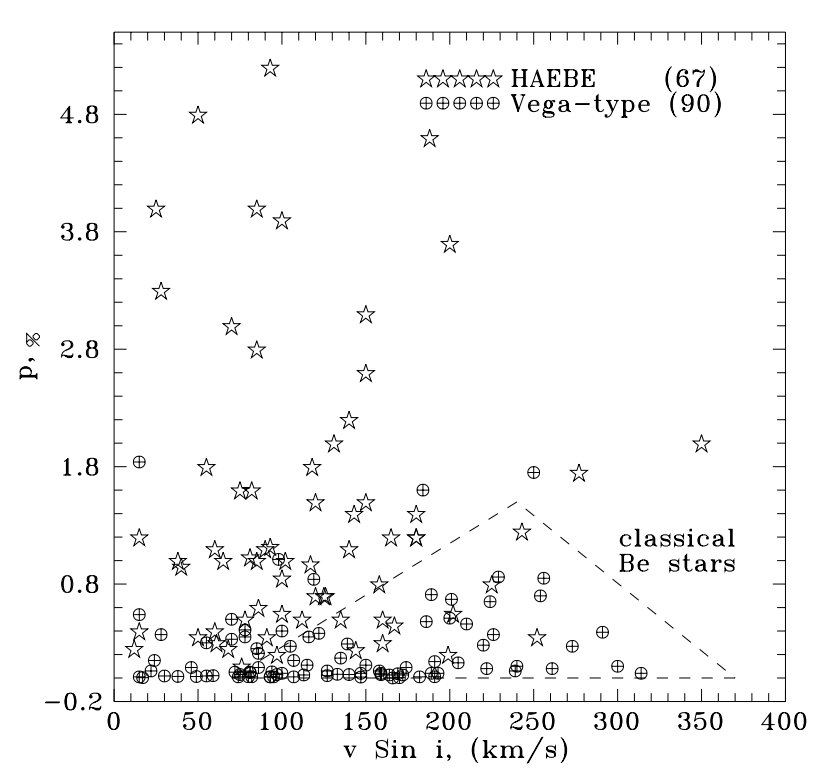

Fig. 13. $\log p / v \sin i$-diagram for HAEBE+Vega-type stars

The important conclusions from this section may be emphasized as follows: most young MS stars and stars in the stage of evolution close to MS exhibit very small levels of intrinsic polarization as well as small (or even absence of) near IR excesses. However some differences between young MS stars and young stars close to the end of the PMS stage of evolution may be seen in the sense that the latter exhibit larger intrinsic polarization and near-IR excesses.

\section{Polarization and projected rotational velocities}

\subsection{Young stars}

The main critical comment to the first paper of Yudin (1988) was that in the case of disk-like distribution of scatterers around the stars (that is at present a general model for the observed behaviour of young stars and many direct evidence of such geometry now exists) no linear correlations between $\log p$ and IR excesses should be observed. The justification for this suggestion is that the polarization is proportional (for optically thin disks) to the value of $\sin ^{2} i$ where $i$ is an inclination angle of the disks to the line of sight. The referees have commented on the existence of a "triangle diagram" rather than a linear correlation. However as follows from Fig. 4, even the significantly larger statistics demonstrate again the pronounced relationship between $\log p$ and $E(V-L)$ for young stars. Note however that at present we don't know which objects have exact pole-on orientation of CS disks for the majority of young stars (maybe with a few exceptions). Even

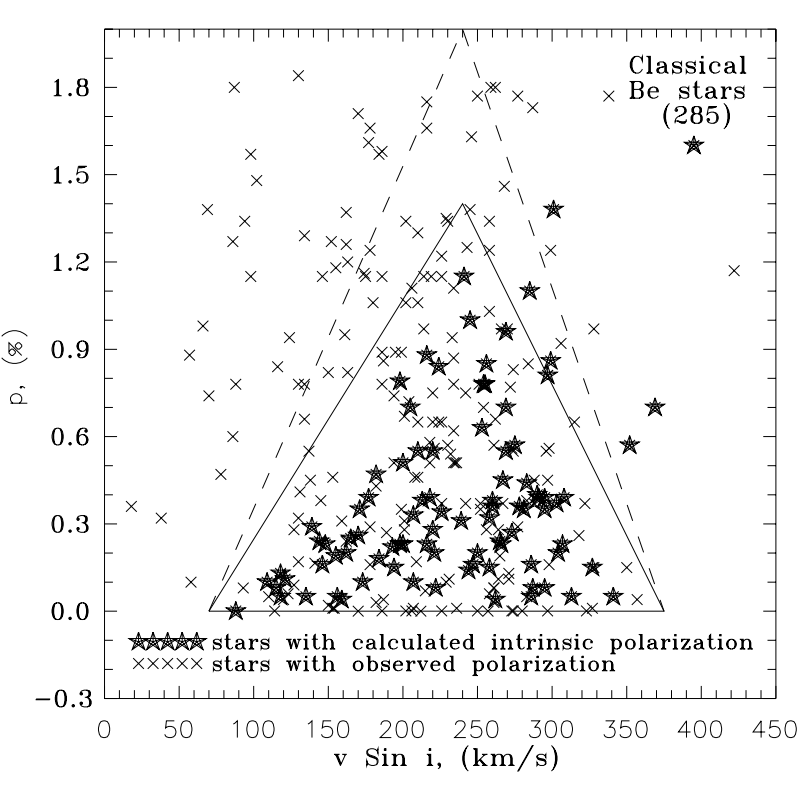

Fig. 14. $\log p / v \sin i$-diagram for classical Be stars

though the $v \sin i$ value is small it is not uniquely determined that the CS disk has a pole-on orientation while some stars may have low rotational velocity themselves. All available data on the rotational velocities for young stars (about 250 objects; see Appendices 1-5) allow us to conclude that no correlation exists between $p_{\text {obs }}$ and $v \sin i$ (see Fig. 13).

Moreover, there is reason to believe that the compression of CS environment takes place along the lines-of force of the interstellar magnetic field (see for example Grinin et al. 1991 and Tamura \& Sato 1989). For the most part the young stars are located in the plane of the Galaxy but the orientation of interstellar magnetic field is mainly colinear with the direction of the axes of spiral arms. In the paper of Andriasyan \& Makarov (1989) the directions of the Galactic coordinates for which the orientation of interstellar magnetic field is perpendicular to the line of sight were separated out. The correlation of these coordinates with those for the stars in our sample shows that about $90 \%$ of the objects lie in those regions. Thus we can reject the suggestion of pole-on orientation of CS disks for most of the selected stars. In addition note that, even for the pole-on orientation of the disks polarization of radiation may take place due to the presence of the rotating dust inhomogeneities in CS shells, or if the disks have no circular symmetry (for example for an ellipse-like envelope and location of a star in one of the focus of this ellipse). In any case even a small departure of $i$ from $0^{\circ}$ gives an ellipse for the projection of the disk on the sky-plane which results in the detectable polarization of radiation (see Discussion in Yudin et al. 1999). Nevertheless, some influence of the 


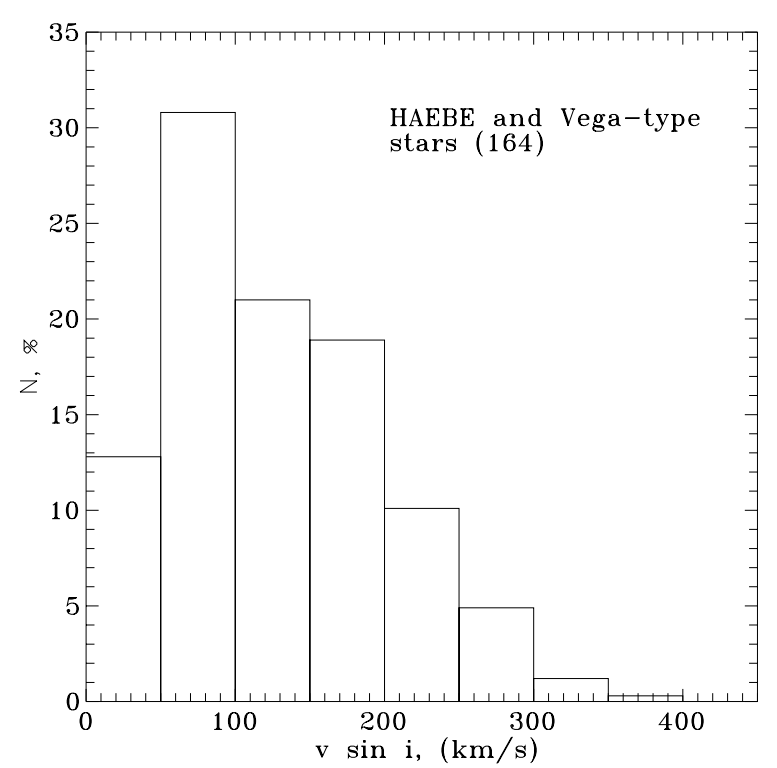

Fig. 15. Histogram of $v \sin i$ values for HAEBE+Vega-type stars

disk's inclination on the observed polarization does occur for individual stars (see for example Yudin et al. 1999). One can suppose that the inclination effect may be pronounced for homogeneous and optically thin disks when the main mechanism of polarization is the scattering in these envelopes and no other sources of polarization exists (such as that of scattering on dust inhomogeneities or condensations revolving around a star).

We may also compare the values of intrinsic polarization and $v \sin i$ for the group of HAEBE stars with Algollike minima (most of them have the similar spectral types around A5). During the photometric minima due to eclipse of nonpolarized stellar light we can detect scattered radiation from their nonspherical dust envelopes. As can be seen in Fig. 12 there is no evidence that stars with larger values of $v \sin i$ show larger values of polarization. However, at present, the list of this type of HAEBE stars is not large enough to draw definite conclusions. It is interesting that large Algol-like minima of brightness are observed for some stars which have respectively small values of $v \sin i\left(60-90 \mathrm{~km} \mathrm{~s}^{-1}\right)$. Discussion on this behaviour will be given in Sect. 7 .

\subsection{Classical Be stars}

It is interesting to compare polarimetric and $v \sin i$ data discussed here for young stars with those for classical Be stars. The correlation between $p$ and $v \sin i$ values for classical Be stars has been investigated by many authors. McLean \& Brown (1978) plotted the values of $p$ versus

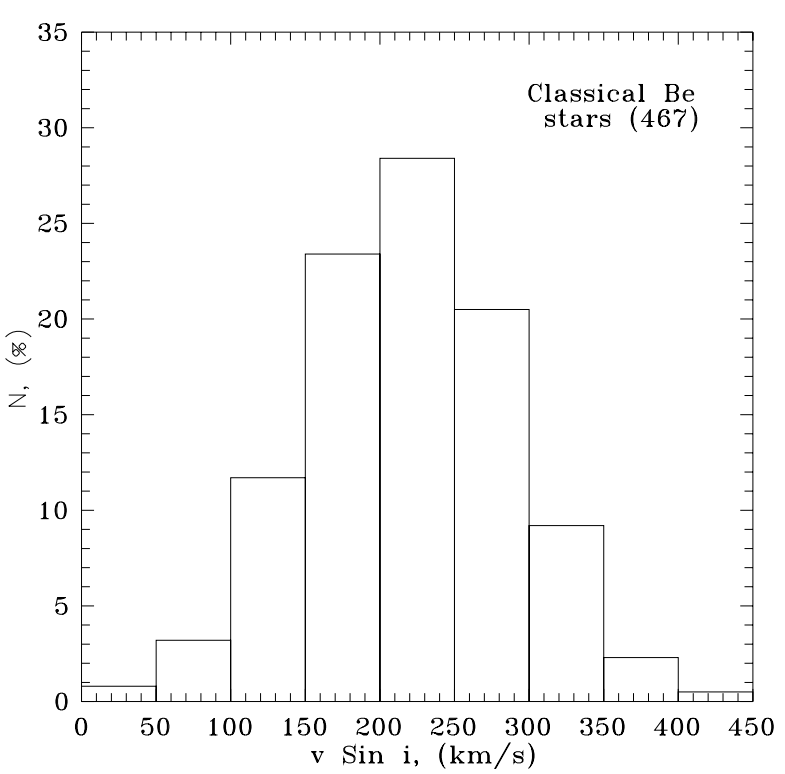

Fig. 16. Histogram of $v \sin i$ values for classical Be stars

$v \sin i$ for a sample of 67 classical Be stars and found a "triangle" distribution (see their Fig. 2). In Fig. 14 we plot the values of polarization versus $v \sin i$ for the larger group of classical Be stars (285 objects). With six times better statistics (compared with previous studies) we can conclude that the relation shows again a "triangle" distribution but not in the same sense as has been discussed by McLean \& Brown (1978). The differences are that the polarization degree has reached maximum values for stars with intermediate values of rotational projected velocities $200 \mathrm{~km} \mathrm{~s}^{-1}<v \sin i<300 \mathrm{~km} \mathrm{~s}^{-1}$ and there are a small portion of stars (mainly Be stars with observed polarization) with large polarization among the stars with relatively low $\left(v \sin i<150 \mathrm{~km} \mathrm{~s}^{-1}\right.$, and very high $(v \sin i>$ $350 \mathrm{~km} \mathrm{~s}^{-1}$, projected rotational velocities. The detailed dicsussion of this behaviour and the results of a statistical study of different observational characteristics of classical Be stars may be found in Yudin (2000).

The investigation of $v \sin i$ distributions was not the initial aim of the present study. However large statistics make it possible to compare the rotational velocity distributions for the groups of stars which are at different stages of evolution, which may be interesting in the context of the present work.

Note that Davis et al. (1983) have noted the difference in the frequency distribution of $v \sin i$ between Herbig Be stars and classical Be stars (but for a small sample). Böhm \& Catala (1995) recently compared $v \sin i$ values for low, intermediate and high-mass HAEBE stars and have estimated the mean projected rotational velocities (MRPV) for 27 HAEBE stars: $v \sin i \approx 105 \pm 35 \mathrm{~km} \mathrm{~s}^{-1}$. 
The histograms of $v \sin i$ distributions for HAEBE+Vegatype stars from our sample and classical Be stars are presented in Figs. 15 and 16 respectively. Despite the fact that both distributions are broad it is reasonably safe to suggest that these distributions diverge considerably. Simple comparison of $v \sin i$ values for stars of different groups leads to the following results: the MPRV for HAEBE+Vega-type stars (164 objects) and classical Be stars are $\approx 105 \mathrm{~km} \mathrm{~s}^{-1}$ and $\approx 220 \mathrm{~km} \mathrm{~s}^{-1}$ (with the standard deviation $80 \mathrm{~km} \mathrm{~s}^{-1}$ and $90 \mathrm{~km} \mathrm{~s}^{-1}$ ) respectively.

One can however note that comparison of the $v \sin i$ distributions is inapplicable for stars having a wide range of spectral classes. However even for 83 Herbig $\mathbf{B e}+\mathbf{B}$ Vega-type stars the MPRV is $\approx 130 \mathrm{~km} \mathrm{~s}^{-1}$ or excluding a few stars which were early classified as classical $\mathrm{Be}-105 \mathrm{~km} \mathrm{~s}^{-1}$. Various statistical tests indicate immediately that the difference between mean values of $v \sin i$ for Herbig $\mathbf{B e}+\mathbf{B}$ Vega-type stars and classical Be stars is statistically significant even at $99 \%$ confidence level. In spite of small statistics the average value of $v \sin i$ for young solar-type stars is smaller than for TT stars (see Appendices 4-5).

As is evident from the theory of stellar evolution, within similar spectral classes the rotational velocities should be smaller for more evolved stars due to the loss of AM (see for example Soderblom et al. 1993 or Dudorov \& Pudritz 1994). However classical Be stars are much more rapid rotators in comparison with young Herbig Be and B Vega-type stars in spite of the former being much more evolved. On the one hand it can be supposed that the increasing of rotational velocities in Be stars takes place due to further contraction and the associated conservation of AM, while a spin down for HAEBE stars is due to net AM loss (by stellar wind) as was supposed by Finkenzeller (1985). But on the other hand the young stars may lose AM by interaction with dust circumstellar shells because classical Be stars have no dust in their environment and this is the primary difference between them. However this leads us to conclude possible differences in initial conditions of formation of Be and young Herbig Be stars because if classical Be stars were surrounded by dust shells in earlier evolutionary phases they would also have low $v \sin i$ values at present (see also discussion in Zorec \& Briot 1997). A detailed discussion of various mechanisms for the redistribution of AM can be found in Brown \& Verschueren (1997).

An important by-product of this study is that the average values of $v \sin i$ differ strongly for classical $\mathrm{Be}$ and young HAEBE + Vega-type stars.

\section{Location of other type stars with IR excesses and polarization of radiation at the $\log p / E(V-L)$ diagram}

For statistical purposes we have compared the location of young stars with the position of the stars which are

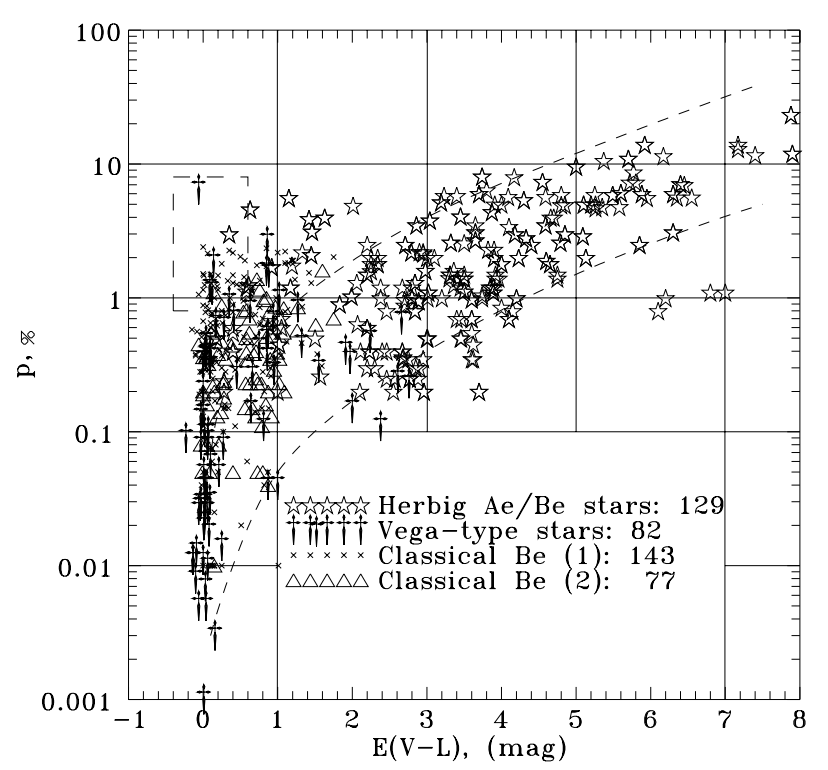

Fig. 17. $\log p / E(V-L)$ diagram for HAEBE stars, Vega-type stars and classical Be stars. The group of classical Be stars (1) contains the objects with observed polarization whereas the group (2) - the objects with calculated intrinsic polarization. The box indicates the position of supergiants from Serkowski et al. (1975)

definitely not young but also show some polarization of radiation.

\subsection{Classical Be stars}

It was previously noted by Yudin (1988) that the classical Be stars (40 objects) did not follow the relation derived for young stars but were distributed in a separate group. To make statistically significant conclusions we collect the data of polarimetry and $V, L$ photometry for a sample of 228 classical Be stars (for detail discussion see Yudin 2000). With few exceptions most of the selected stars are dwarfs that allow us to compare their behaviour with that of HAEBE stars. The relation between $\log p$ and $E(V-L)$ for classical Be and HAEBE stars is shown in Fig. 17. It is safe to say that most classical Be stars are largely concentrated in the region of small IR excesses $0^{\mathrm{m}}<E(V-L)<1^{\mathrm{m}}$ while their polarization varies from very low values up to $\approx 2 \%$. The correlation between $\log p$ and $E(V-L)$ for Be stars is weak or even absent and most of them are concentrated well away from the above derived dependence for young HAEBE stars. It is well known that the polarization of Be stars originates from scattering of free electrons in a CS gaseous shell, as well as the excess in near IR, and even at $12 \mu \mathrm{m}$ is mainly caused by freefree emission (see Waters \& Marlborough 1992) and is not 
due to the thermal emission from CS dust. Therefore, the nature of IR excesses and polarization in classical Be stars and HAEBE stars is quite different. We are currently collecting data on optical polarization, near-IR excesses and projected rotational velocities for a sample of $\approx 650$ classical Be stars and we are planning to publish a detailed study in the nearest future (Yudin 2000).

\subsection{Red giants and supergiants}

Another group of stars with IR excesses and polarization is red giants and supergiants (hereafter RSG). The importance of the comparison of their polarimetric behaviour with that observed for young TT stars is that the objects in both classes are surrounded by dust shells, have approximately the same spectral classes but are on a different stage of evolution. RSG are post-MS objects and they are located on the diagram near $E(V-L) \approx 0$ with a considerable scatter in the polarization values (up to $4 \%$, see Fig. 3). It is well known that for most RSG the dust envelopes are optically thin $\tau \leq 0.1-0.2$ (Dyck et al. 1971). There is a consensus now that the intrinsic polarization of RSG originates due to scattering by nonradially oriented dust grains in the stellar envelope. In this case linear polarization appears even if the stellar envelope is spherical and even for small optical depth (resulting in small near IR excesses). This suggestion is strongly supported by the detection of high value circular polarization in some RSG (up to $p_{\mathrm{c}} \approx 0.5 \%$ ). Comparison of the location of TT stars and RSG on the diagram leads to some interesting conclusions:

a) Most TT stars are located outside the box occupied by RSG which indicates different physical conditions in their CS shells (for instance, significantly larger optical depth in CS shells of TT stars);

b) A small fraction of TT stars $(\approx 20 \%)$ are located close to the position derived for RSG and for some of them we may suppose the important contribution of the scattering by nonspherical dust grains to their intrinsic polarization (see also Lucas \& Roche 1998). This fact has been corroborated by the detection of circular polarization in some TT stars (Bastien et al. 1989). Note however that the level of circular polarization in TT stars is generally lower $\left(p_{\mathrm{c}}<0.1 \%\right)$ than that for RSG. At present circular polarization has been detected for only a few HAEBE stars and on the level lower than that for TT stars. Thus a slight difference in position of HAEBE and TT stars and respectively larger scattering of the data points for TT stars on the diagram may also occur due to the presence of nonspherical oriented dust grains in CS shells of some objects.

\subsection{Main sequence stars in solar neighborhood}

The next group is MS stars in solar neighborhood and 68 stars from Leroy's (1993) catalogue within 50 pc from the Sun with available $L$-band photometry and $p / \sigma p>2$ were selected. It is believed that for most of these stars the interstellar component of polarization is negligible. As follows from Fig. 3, all selected stars are largely concentrated in a small region of the diagram around $E(V-L) \approx 0^{\mathrm{m}}$ and in the range of polarization $0.01 \%<p<0.1 \%$, i.e. in the same region as young solar-type stars and some Vegatype stars. This is a side benefit on the small polarization of MS stars.

\subsection{Early-type supergiants}

Finally, we consider the Serkowski et al. (1975) catalogue. About 120 stars for which the data on $L$-band photometry were found in CIO5 (Gezari et al. 1999) have been selected. Most of them have MK-classification and are supergiants of early spectral type which allows us to exclude the suggestion of their possible youth. As follows from Fig. 2 most of these normal stars are clustered close to the value of $E(V-L) \approx 0^{\mathrm{m}}$ in the range of polarization $0.3 \% \leq p \leq 8 \%$. This fact suggests that these stars have no near IR excesses (and no hot dust in their CS environment) and all observed polarization has an interstellar origin. If the component of interstellar polarization is removed from the observed values, these MS stars will be located in the lower left side of the diagram discussed here. In any case their position on the diagram is very different from the dependence obtained for young stars which gives additional support to the use of this diagram as a selection criteria for young stars.

The conclusion of this section is that the regions of the diagram occupied by stars which are not young are distinguishable from those occupied by young stars of different spectral types (see Fig. 4). All the above might be considered as a visual demonstration that the diagram can be used for the selection of stars with hot CS dust (and most of them might be young objects).

\section{Polarization and scattering light}

Due to the multicomponent structure of CS shells around young stars several sources of observed polarization exist. These are: - scattering of radiation in nonspherical disklike dust shells; - Thomson scattering in CS gaseous shells; - Thomson scattering in gas jets; - scattering on gas and dust inhomogeneities rotating around a star etc.

It is important that large IR excesses $(>1 \mathrm{~m} .5)$ at $3.5 \mu \mathrm{m}$ cannot be explained by free-free and free-bound emission. This is in good agreement with the data for classical Be stars (most of them show near IR excesses $<1^{\mathrm{m}}$ ). Thus the main source of IR excesses in young stars is the 
absorption and re-emission of radiation by dust grains. Because strong correlations between polarization and IR colour excess $E(V-L)$ exist for the majority of young stars we suppose that the main source of polarization in young stars is the same as for near IR excesses i.e. CS dust. Therefore we should not consider here the mechanisms of polarization which are connected with CS gaseous shells. In the framework of this assumption the observed polarization in young stars can be described as a vector sum of several components:

$P_{\mathrm{obs}}=P_{\mathrm{is}}+P_{\mathrm{CSdisk}}+P_{\mathrm{CSinhom}}$

where $P_{\text {is }}$ - is the interstellar component of polarization, $P_{\text {CSdisk }}-$ is polarization arising in the nonspherical dust envelope and $P_{\mathrm{CSinhom}}$ - is polarization due to scattering CS dust inhomogeneities. As a first step we assume that $P_{\text {CSinhom }}$ does not affect strongly the value of observed polarization. Although this component of polarization is rather small, as will be shown later, the presence of these inhomogeneities in CS shells is an important factor that may result in large polarimetric variations indirectly. We also exclude from our study the interstellar component of polarization.

Grinin et al. (1995) in the context of the observational behaviour of UXOrs have discussed two possibilities for the intrinsic polarization in the framework of the dust model, namely optical dichroism of CS nonspherical grains, and the scattering of stellar radiation in CS dust shells. They noted that in the first case a linear dependence must be observed between the amplitude of brightness variations and polarization degree:

$P_{\text {int }}=\alpha \times \Delta m$

where $\alpha$ - is the factor dependent on the degree of alignment of the dust particles in a SC cloud. In the second case, the dependence is quite different and has the form:

$P_{\text {int }}=P_{\text {int }}^{0} \times 10^{0.4 \Delta m}$.

As may be inferred from observations of UXOrs as well as from our analysis, the second case is much more acceptable as an explanation for the behaviour discussed in the present study.

In fact, $P_{\text {int }}^{0}$ is dependent on the amount of hot dust in CS shells and the geometry of the envelopes. Note that it is impossible to explain the observed polarization in all young stars assuming that the dust envelopes have a spherical shape. Therefore we will consider the disk-like envelopes.

Let us consider two components $\left(P_{\text {int }} \sim P_{\text {int }}^{0}\right.$ and $P_{\text {int }} \sim 10^{0.4 \Delta m}$ ) in Eq. (7) separately. In the general case polarization for the disk-shaped envelope can be described by the following formula (see Dolginov et al. 1995):

$P_{\text {int }}^{0}(\%) \approx 300 / 16|\bar{b}| \times f(d / R) \times \tau \sin ^{2} \theta$

where $\tau$ - is the optical thickness of the envelope, $f(d / R)$ - a function dependent on the ratio of envelope geometrical thickness to the radius, $i$ the inclination angle of the disks to the line of sight and $\bar{b}=1 / q=1-\sigma_{\mathrm{a}} / \sigma_{\mathrm{t}}\left(\sigma_{\mathrm{a}}\right.$

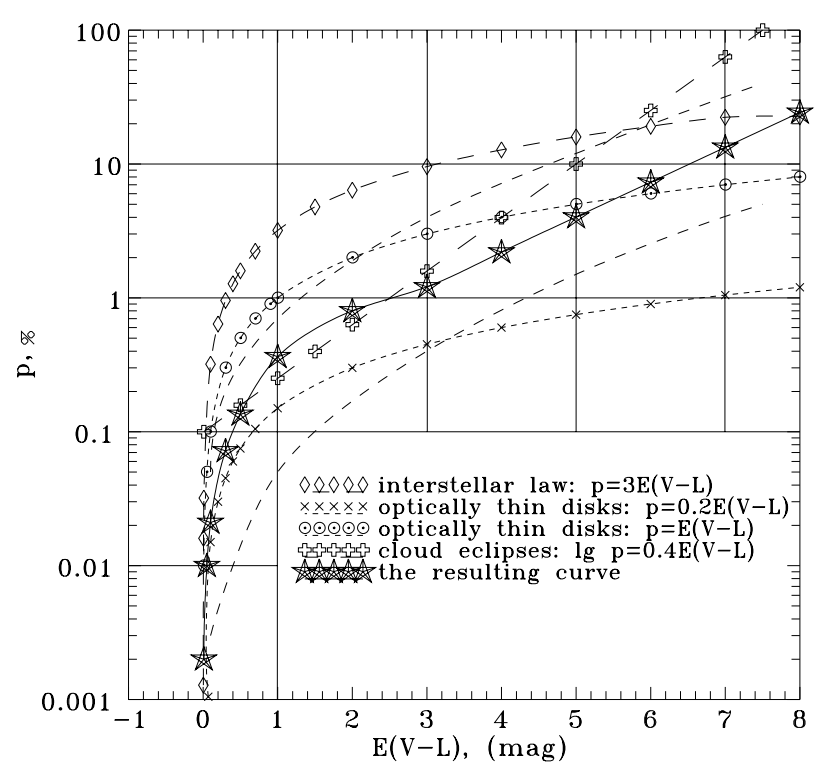

Fig. 18. $\log p / E(V-L)$ relations for different sources of polarization

and $\sigma_{\mathrm{t}}$ are absorption and scattering cross-sections). Let us re-construct this formula in terms of $E(V-L)$. Using the canonical equations $A_{V}=1.086 \tau_{V}$ and the relation between $E(B-V)$ and $E(V-L)$ the optical thickness $\tau_{V} \approx 0.98 \times E(V-L)$. Thus

$P_{\text {int }}^{0}(\%) \approx 300 / 16 \times f(d / R) \times E(V-L) \times \sin ^{2} \theta$.

Numerical values for $f(d / R)$ are tabulated in Dolginov et al. (1995). For favorable geometry and $\sin ^{2} \theta=1$

$P_{\mathrm{int}}^{0}(\%) \approx E(V-L)$

or for the intermediate case

$P_{\text {int }}^{0}(\%) \approx 0.2 \times E(V-L)$.

The relations $(10,11)$ are plotted in Fig. 18. Note that these relations cannot explain our dependence adequately. Moreover these relations are valid only for the optically thin case which corresponds to $E(V-L) \leq 1^{\mathrm{m}}$. Note however that in the case of the presence of optically thick dust condensations in the midplane of the envelope instead of the optically thick narrow dust disk, the average optical thickness of this envelope may reach the value of $\tau \approx 1$ at values of $E(V-L) \approx 3^{\mathrm{m}}$ or even larger. Incidentally, the relation (10) is in agreement with the data for classical Be stars for which the polarization is due to the scattering of free electrons in nonspherical optically thin disk-like CS gaseous shells. The relations $(10,11)$ are also valid for some TT stars and describe well the position of most Vega-type and post HAEBE stars. This fact is in good agreement with the above suggestion on the presence of optically thin and homogeneous disks around them. 
Let us consider now the second term of Eq. (7) assuming $P_{\text {int }}^{0} \leq 1$ (this corresponds to the observed values for UXOrs in their bright states).

Because of the assumption on nonvariable IR fluxes we can re-write $\Delta m \approx \Delta(V-L)=E(V-L)$ and

$P_{\text {int }}(\%)=10^{0.4 E(V-L)}$

or

$P_{\text {int }}(\%)=\mathrm{e}^{E(V-L)}$.

This relation is also shown in Fig. 18. Note that as is evident from the present statistical study the polarization is proportional to near IR excess $E(V-L)$ in the form $P \sim$ $\mathrm{e}^{k_{1} E(V-L)}$ or $P \sim 10^{k_{2} E(V-L)}$. However the coefficient $k$ in our relation (3) differs from those in Eqs. $(12,13)$. To make the coefficients consistent we must assume that the extinction curve for dust clouds/condensations is different from that of the standard curve. Put another way the extinction in near IR must be higher: $\tau_{V} \approx 0.6 \times E(V-L)$ which corresponds to the ratio $A_{L} / A_{V} \approx 0.2-0.4$ i.e. the extinction curve is flatter than the interstellar one (see also Mitskevich 1995). This partly nonselective extinction can occur only due to the presence of large dust grains in clouds from which stellar radiation is obscured. Rostopchina et al. (1997) noted recently that the modelling of the behaviour in UXOrs requires the minimum size of the dust particles to be about ten times larger than that of the interstellar matter. They have discussed this fact in terms of the growth of particles in these kinds of objects and noted that these particles are still smaller than in the "old" protoplanetary disks of Vega-type stars. Simultaneous optical and IR photometry for some UXOrs which was carried out by Hutchinson et al. (1994), clearly indicates the decreasing of IR fluxes during a visual fade with the ratio that is in excellent correspondence with $A_{L} / A_{V} \approx 0.3$. According to Chini et al. (1990) the minimum size of the dust particles in CS shells around Vega, $\alpha$ PsA and $\epsilon$ Eri is $a_{\min }>20 \mu \mathrm{m}$. Similar conclusions were recently made for HD 45677 by de Winter \& van den Ancker (1997) who suggested that photometric variations appeared in the star due to obscuration by CS condensations with large $(>1 \mu \mathrm{m})$ dust grains. Some evidence for unusually large grains in front of some Orion stars (most of them are in our list of post HAEBE and Vega-type stars, see Appendices 1-2) has been discussed by Breger (1977) and Breger et al. (1981). Moreover, there is much evidence that the wavelength dependence of polarization in most young stars diverges considerably from that determined by the Serkowski law, namely in the sense that polarization in the red and near IR is often higher that might be expected for standard interstellar grains (see Tamura \& Sato 1989; Vrba et al. 1979; Garrison \& Anderson 1978).

Taking into account the above discussion the expression for the degree of intrinsic polarization for the majority of young stars has the form:

$P_{\text {int }}(\%) \approx 0.2 E(V-L) \times \mathrm{e}^{0.6 E(V-L)}$ for the region of $0^{\mathrm{m}}<E(V-L)<1^{\mathrm{m}}$ or $\leq 3^{\mathrm{m}}$ and

$P_{\text {int }}(\%) \approx 0.2 \times \mathrm{e}^{0.6 E(V-L)}$

for the region of $3^{\mathrm{m}} \leq E(V-L)<8^{\mathrm{m}}$.

Note that the expression derived above corresponds well with the statistical dependence (see Fig. 18). It is also important that even for optically thin disk-like dust envelopes the polarization degree can be greater than $\sim 6 \%$ if the stellar radiation is screened (see series of papers by Daniel 1980a, 1980b, 1982 or Voshchinnikov \& Karjukin 1994).

Finally, the change in the average line inclination should be caused by multiple scattering if the optical thickness of the shell $\tau>1$ (this may occur in some young stars). This leads to a decrease in the fraction of scattering radiation and decreasing of observed polarization (Voshchinnikov \& Karjukin 1994).

In addition note that the conclusion of the existence of large dust particles in CS envelopes of young stars, which follows from the analysis of the behaviour of young stars on the diagram discussed here, is beyond the reach of classical $p-\Delta m_{\text {visual }}$ diagrams. Thus, the diagram discussed here is more informative.

\section{Some astrophysical aspects of the $\log p / E(V-L)$ diagram}

At the moment there is consensus on the changes in the masses of CS shells on the different stages of evolution. For detail discussions see for example:

1. Zuckerman \& Beklin (1993) (the mass of dust in small grains is a sharply decreasing function of time from pre-MS to MS stars due to the coagulation of grains to planetesimal bodies);

2. Fuente et al. (1998) (there is a progressive dispersion of the dense gas associated with HAEBE stars in their evolution to the MS and there is good correlation between the ages of HAEBE stars and the observed dispersal on a large scale of CS material;

3. Coulson et al. (1998) (classical TT and HAEBE stars contain more dust $\left(10^{-5}-10^{-2} M_{\odot}\right.$ and $10^{-2}-10^{0} M_{\odot}$ respectively) than Vega-type systems $\left(10^{-7}-10^{-4} M_{\odot}\right)$.

On the other hand the evolutionary changes is CS masses are not so evident on a relatively short time-scale. Mannings \& Sargent (1997) declared that the masses of the dust disks around Herbig Ae stars have changed little during their 5-10 Myr lifetime. Nata et al. (1997) claimed that "no systematic differences in $M_{\mathrm{CSD}}$ (CSD - circumstellar dust) between classical HAEBE stars and young stars with Algol-like minima, as well as no statistically significant correlations between $M_{\mathrm{CSD}}$ and stellar age is found, and the amplitude of photometric variability appears to be independent of age". They also 
noted that UXOr phenomena do not characterize a more evolved environment.

Thus we conclude that the changes in the envelope masses are clearly evident for objects which are at the significantly different stages of pre-MS evolution and we should try to consider all the above mentioned suggestions in the discussion below.

The next important point is to make a comparison of the polarization and photometric variability. According to Grady et al. (1996) "all of the stars which show photometric variability larger than $1^{\mathrm{m}}$ are stars with accreting gas detection and the accretion activity seen toward $\beta$ Pic began during the earlier PMS evolution of the star and its CS disk". Ancker et al. (1998) noted that no HAEBE stars with small IR excesses at 3.6 and $12 \mu \mathrm{m}$ show strong photometric variability, whereas those with larger excesses show a large spread in $\delta H_{\mathrm{p}}$. They have also noted that $\beta$ Pic like systems with usually smaller IR excesses show a range in $H_{\mathrm{p}}$ less than $0^{\mathrm{m}} .1$.

Analysis of polarimetric and photometric characteristics of early-type stars from our sample indicates that a few different groups of objects may be distinguished.

1. Objects with large, non-variable (on a short time scale - days) polarization and large near IR excesses $E(V-L)>5^{\mathrm{m}}$. These objects mainly show a high level of polarization $(p>5 \%)$ over long periods. Large photometric and polarimetric variability in these objects may be observed but also on a large time scale;

2. Objects with intermediate near IR excesses $5^{\mathrm{m}}>$ $E(V-L)>3^{\mathrm{m}}$ which exhibit large polarimetric variability on a short time scale (days) $(\Delta p>3 \%)$ and show essentially small polarization over long periods $(p \approx 1 \%)$. Variations in photometry are also significant (up to $2^{\mathrm{m}}-3^{\mathrm{m}}$ );

3. Objects with small near IR excesses $3^{\mathrm{m}}>E(V-L)>$ $1^{\mathrm{m}}$ and non-variable polarization $(p \approx 1 \%$ and $\Delta p<$ $1 \%)$. Photometric variability for these stars are rather small and does not exceed 0 m.5;

4. Objects with small near IR excesses $E(V-L)<1^{\mathrm{m}}$, small polarization $(p<0.5 \%)$ and small photometric variability $\Delta m \ll 1^{\mathrm{m}}$, or even non-variable in photometry and polarization.

We suppose that this differentiation is caused by the changes in structure and geometry of dust circumstellar shells around the stars and these changes are caused by evolution of the dust circumstellar environment.

It is interesting that some HAEBE stars with Algollike minima of brightness and low values of $v \sin i$ show large photometric and polarimetric variations. If the value of $v \sin i$ in fact reflects the inclination of CS dust disks, the opening angle of these disks should not be small to explain the observed eclipses. For UXOrs' disks the opening angle was estimated to be of the order $35^{\circ}$ (see Nata et al. 1999). In the case of the relatively large inclination angle the dust inhomogeneities which are responsible for the eclipse should have a size of the order of a stellar surface. However, these sizes of dust inhomogeneities are much less than those we can expect for younger objects (taking into account the time scale of their photometric and polarimetric variations). Conceivably, the formation of compact dust inhomogeneities in CS environments must reflect a definite stage of CS shell evolution. Ancker et al. (1998) noted that the patchy dust clouds are only present during the pre-MS evolution of a star. They either vanish or become more homogeneous when a star has reached the ZAMS. On the other hand, for extreme young IR sources (such as L1551 IRS5: Men'shchikov \& Henning 1997, HL Tau: Men'shchikov et al. 1999, GSS 30: Chrysostomou et al. 1997 or R Mon: Magnier et al. 1999) the opening angle of non-spherical dust envelopes was estimated to be of the order $>50^{\circ}$ or even $\approx 90^{\circ}$ whereas for $\beta$ Pic the opening angle was estimated to be $10^{\circ}$ (Artymowicz et al. 1989) or even $2^{\circ}$ (Chini et al. 1991).

To explain the observational behaviour in young stars we can suggest a two-component structure for CS dust shells: a large optically thin but geometrically thick disklike envelope + a narrow optically thick (sometimes accretion) disk. Another possibility is to assume that the concentration of the dust particles significantly increases to the midplane of the disk-like envelope with the presence of optically thick dust condensations preferably in the midplane instead of the homogeneous and optically thick accretion disks. These two assumptions in principle may characterize different stages of CS shell evolution and/or reflect the differences in the CS shell configuration in TT and HAEBE stars. There is much evidence that the second case is more acceptable for classical HAEBE stars (see Discussion in Mitskevich 1995).

The important conclusion which emerge from the above are as follows:

For the stars with geometrically thick dust disk-like shells which screen most of the nonpolarized stellar radiation we observe mainly scattered radiation from their nonspherical CS envelopes (i.e. high level of observed polarization). These geometrically thick disks are homogeneous i.e. not many clouds or holes exist in their circumstellar envelopes, or these clouds are very large (like that for R Mon, V 376 Cas, MWC 349 etc. see for example Yudin 1996; Matsumura et al. 1999). Therefore large polarimetric and photometric variations for these objects are apparent in a time scale of tens of days and is not obvious for shorter periods (for a similar discussion see Matsumura et al. 1999 [for R Mon]). For UXOrs the disks are still relatively geometrically thick but their width is less than those for younger objects. These disks (or nonspherical envelopes) are rather optically thin but contain numerous optically thick condensations. Besides, the dust cloud size is comparable to the stellar radius. For these objects significant variations in polarization and photometry occur over a few days but over longer periods they show a low level of polarization and a small amplitude photometric 
variability. At the later stage of evolution the young stars exhibit geometrically thin disks and dust inhomogeneities with the size significantly less than the stellar surface. These disks and dust condensations cannot screen stellar radiation much so the observed polarization is small (even if the small-amplitude Algol-like minima are observed, see Vieira et al. 1999 for HD 100546). On the other hand the CS disks around Vega, Fomalhaut and $\beta$ Pic are still extended but geometrically and optically thin and homogeneous therefore no significant polarization occurs and no significant light variation exists in these objects (see for example Lecavelier des Etangs et al. 1997).

It can be said with confidence that possible evolutionary changes in the geometry of CS disks are in good agreement with observational features, at least in early-type young stars.

The evolution of disks from optically and geometrically thick to optically and geometrically thin has been also suggested for TT stars by many authors (see for example Strom et al. 1989).

First we compare the polarization values for TT stars with "active" and "passive" CS disks. In terms of the classification of Miyake \& Nakagama (1995) these disks can be distinguished by different accretion rate $\dot{M} \geq$ $10^{-8} M_{\odot} \mathrm{yr}^{-1}$ and $\dot{M} \leq 10^{-9} M_{\odot} \mathrm{yr}^{-1}$ respectively. Miyake \& Nakagama (1995) have discussed the possible evolutionary sequence from "active" to "passive" class I disks. Using the available data on the polarimetry of TT stars, it is possible to compare the distribution of the polarization degree and near IR excesses for the objects with different kinds of CS disks.

In spite of the low statistics we may note the changes in the polarization degree from $\approx 2.5 \%$ for "active disks" through $\approx 1.1 \%$ for class I "passive" disks to $\approx 0.5 \%$ for class IV and II disks. Slightly better statistics for near IR excesses show the same decrease in $E(V-L)$ respectively: "active" disks $-E(V-L) \approx 2$ m 15 , class I $E(V-L) \approx 1^{\mathrm{m}} \cdot 60$, class IV $-E(V-L) \approx 1^{\mathrm{m}} .30$, class II $E(V-L) \approx 1^{\mathrm{m}} .15$ and class III $-E(V-L) \approx 0 \mathrm{~m} \cdot 2$.

The main conclusion from the above is the following: most TT stars which according to Miyake \& Nakagama (1995), are accompanied by "active" CS disks, show significantly larger polarization and near IR excesses and possibly average polarization and IR excesses decrease with the disk's evolution.

At the latest stage of a TT phase (so-called weak-line TT stars) the disks become optically thin (low polarization and low near IR excesses), in good agreement with the results of Bastien et al. (1996) who have noted that "weak TT stars exhibit a small polarization averaging $0.7 \%$ and with no $p$ in excess of $2.4 \%$ while classical TT stars exhibit an average polarization of $1.6 \%$ with a high polarization tail".

\section{Polarization and SED in the far IR of young stars}

According to many authors (see for example Adams et al. 1987) young stars show a random shape of their SEDs in far IR from that with flat or rising IR spectra through two-component IR SEDs to that with decreasing IR spectra. There is no doubt at present that these changes in the shape of the SED are caused by evolution of dust CS shells. Hillenbrand et al. (1992) discussed the evolutionary changes of the SEDs for HAEBE stars from their group II (with flat or rising IR spectra) through group I to group III (with small IR excesses). Malfait et al. (1998) have studied the more evolved HAEBE stars (45 objects), that arguably are evolving towards Vega-type stars. Most of the objects in their study may be classified as the group I objects of Hillenbrand (1992) but 11 stars from their sample lie on the $(J-H) /(H-K)$ diagram closer to the MS region. They discussed a possible evolutionary scenario in the changing of the SEDs from the embedded sources (group II of Hillenbrand (1992) through the stars with broad IR excesses and HAEBE stars with a composite excess (group I of Hillenbrand (1992) to Vegatype stars. A possible evolutionary sequence among young stars has been proposed recently by Yudin et al. (1999) as follows: "Herbig Ae/Be stars (i.e. pre-MS stars) $\Longrightarrow$ Vega-like stars with near-IR excess (i.e. young stars near the end of the pre-MS evolutionary phase) $\Longrightarrow$ Vega-like stars without near-IR excess but with some far-IR excesses (i.e. young MS stars) $\Longrightarrow$ MS stars without nearand far-IR excesses". If the changes in dust structure of CS environment during the evolution are real, the changes in average polarization should be observed simultaneously. Using even small statistics Hillenbrand et al. (1992) noted that the objects in their groups I and III generally show smaller net polarizations $(p<2 \%)$, than the objects in group II $(p>5 \%)$. This behaviour will be investigated below (using larger statistics).

Like Malfait et al. (1998) we separate young stars from our sample with different types of IR SEDs and plot this SEDs sequence in Fig. 19. The results of our selection are tabulated in Table 4. We have not constructed here SEDs for all stars from our sample but have used SEDs which were available in the literature (Hillenbrand et al. 1992; Malfait et al. 1998; Mannings 1994; Gauvin \& Strom 1992; Grinin et al. 1991; Miyake \& Nakagama 1995). Thus we compare the values of $p$ and SEDs for about 100 young stars.

As can be seen immediately from Table 4 there is a definite sequence of changing in polarization values from group 1 (with flat or rising IR spectra) $-p \approx 8 \%$ through group 2 (with decreasing IR spectra) $-p \approx 1.7 \%$, to groups 3 and 4 (with small IR excesses) $-p \approx 0.8 \%$ and $0.4 \%$ and further to group 5 which contains the objects which are close to the MS $-p<0.1 \%$.

Note that without exception all stars in group 1 are sources of active outflow. In spite of the fact that early 
Table 4. Sample of stars with different type of SEDs in near and far IR (1, 2, 3, 4, 5 - according to Fig. 19). The last line in the table indicates the number of stars in each class and an average value of polarization $\bar{p}$

\begin{tabular}{|c|c|c|c|c|c|c|c|c|c|c|c|}
\hline \multicolumn{2}{|l|}{1} & \multicolumn{2}{|l|}{2} & \multicolumn{4}{|c|}{3} & \multicolumn{2}{|l|}{4} & \multicolumn{2}{|l|}{5} \\
\hline name & $p, \%$ & name & $p, \%$ & name & $p, \%$ & name & $p, \%$ & name & $p, \%$ & name & $p, \%$ \\
\hline V 376 Cas & 23.4 & HD 259431 & 1.0 & HD 98922 & 0.2 & HD 179218 & 0.4 & HD 17081 & 0.37 & Vega & 0.005 \\
\hline LkH $\alpha 198$ & 1.9 & HD 50138 & 0.9 & HD 104237 & 0.25 & HD 142666 & 0.5 & HD 158643 & 0.46 & HD 216956 & 0.007 \\
\hline Elias 1 & 4.7 & RR Tau & 0.4 & V856 Sco & 0.5 & HD 163296 & 0.2 & HD 41511 & 0.55 & HD 4881 & $<0.1$ \\
\hline R Mon & 13 & HD 150193 & 5.2 & HD 139614 & 0.15 & HD 190073 & 0.4 & HD 141569 & 0.10 & & \\
\hline $\mathrm{R} \mathrm{CrA}$ & 6 & MWC 297 & 2 & HD 132947 & 1.3 & AB Aur & 0.5 & HD 169142 & 0.3 & & \\
\hline DG Tau & 5 & MWC 1080 & 2.6 & HD 34282 & 0.11 & VV Ser & 1.0 & HD 52721 & 1.25 & & \\
\hline $\mathrm{LkH} \alpha 25$ & 3.5 & TY CrA & 1.0 & HD 31648 & 0.25 & HD 35929 & 0.11 & HD 58647 & 0.2 & & \\
\hline $\mathrm{T} \mathrm{CrA}$ & 2.9 & NX Pup & 0.7 & HD 36112 & 0.25 & HD 53367 & 0.7 & HD 37490 & 0.45 & & \\
\hline RCW 34 & 5.6 & HK Ori & 1.8 & HD 37258 & 0.3 & $\mathrm{LkH} \alpha 208$ & 2.8 & $+46^{\circ} 3471$ & 0.7 & & \\
\hline V 1686 Cyg & 5.4 & LkH $\alpha 234$ & 1.4 & HD 37806 & 0.7 & $+40^{\circ} 4124$ & 1.2 & $+41^{\circ} 3731$ & 0.35 & & \\
\hline KK Oph & 4 & HD 250550 & 1.1 & HD 144432 & 0.35 & LkH $\alpha 218$ & 1.6 & TW Hya & 0.15 & & \\
\hline HL Tau & 11 & XZ Tau & 1.6 & HD 97048 & 2.2 & HD 37411 & 0.25 & & & & \\
\hline PV Cep & 14 & T Tau & 1.2 & HD 95881 & 1.5 & VZ Cha & 1 & & & & \\
\hline VV CrA & 3.8 & MWC 137 & 6 & SU Aur & 0.4 & $+65^{\circ} 1637$ & 1.2 & & & & \\
\hline & & V380 Ori & 1.2 & AK Sco & 0.5 & HD 200775 & 0.95 & & & & \\
\hline & & AA Tau & 1.5 & CQ Tau & 1.0 & RY Lup & 0.5 & & & & \\
\hline & & CX Tau & 0.65 & CO Ori & 1.6 & WW Vul & 0.5 & & & & \\
\hline & & T Ori & 1.5 & BP Tau & 0.3 & DF Tau & 0.4 & & & & \\
\hline & & DN Tau & 0.7 & UZ Tau & 0.75 & V773 Tau & 0.11 & & & & \\
\hline & & DS Tau & 0.8 & V819 Tau & 1.3 & V826 Tau & 0.95 & & & & \\
\hline & & Haro 1-1 & 2.9 & V827 Tau & 0.6 & V830 Tau & 0.6 & & & & \\
\hline & & UX Ori & 2.0 & & & & & & & & \\
\hline 15 & $7.7 \%$ & 25 & $1.7 \%$ & & 48 & $0.7 \%$ & & 12 & $0.4 \%$ & 3 & $\leq 0.1 \%$ \\
\hline
\end{tabular}

stages of evolution are also characterized by high value of accretion rate, the existence of active matter outflow may well counteract the accretion. During further evolution of CS shells (changes in SED from group 1 to group 4) CS disk-like envelopes being compressed along a lineof-force of magnetic field become more flattened and the accretion becomes more important mechanism for perturbation of dust in CS environment. Simple comparison of the sources from Table 4 with the lists of objects from Grady et al. (1996) indicates that some of the objects in our group 2 and 3 show evidence of both the matter outflow and accretion. At this stage of evolution the changes in polarization may occur for a few reasons: the decreasing of amounts of hot dust in CS disks, the compression of dust disks into very narrow structures which is unfavorable for polarization and possible increasing of optical depth around the disk's midlplane which results in multiple scattering and depolarization effects. Note also that all stars with Algol-like minima (such as those stars in the HAEBE and TT groups) have their SEDs around class 3 objects which is in good agreement with the scheme of evolution depicted above, particularly with respect to compression of CS disks. All UXOrs show clear evidence of a disk accretion (Nata et al. 1997) as well as many other stars in groups 3 and 4.

Finally, the evolution of CS disks from group 4 to group 5 and further, leads to a strong decrease in both near and far IR excesses. However the decreasing of near IR ex- cesses occurs more rapidly (see Malfait et al. 1998) because no matter outflow exists at this stage. All prototypes of Vega-excess stars show evidence of accretion but no matter outflow. At this stage of evolution CS disks have very small optical depth, are very narrow and in addition some fraction of CS dust may coagulate into the large bodies. All of this provides unfavorable conditions for polarization (clearly indicated in Table 4 and as follows from all the above discussion).

We emphasize that the above discussed scheme of CS evolution is only a simple sketch and no numerical calculations have been made here. For detail theoretical discussion of this subject see for example Miyake \& Nakagama (1995) and Myers et al. (1998).

We must also emphasize that the changes in polarization and IR excesses may not be caused directly by the age of a star itself and rather reflect the evolutionary changes in CS disk-like shells. The link between the age of a star and the stage of the evolution of it's CS shell certainly exists but this link can be very complicated and depends on many factors (the mass of a star, the rotational velocity, binarity etc.).

\section{Conclusions}

We have presented the results of statistical study of correlations between polarization and photometric behaviour for a representative group of young stars of various types. 


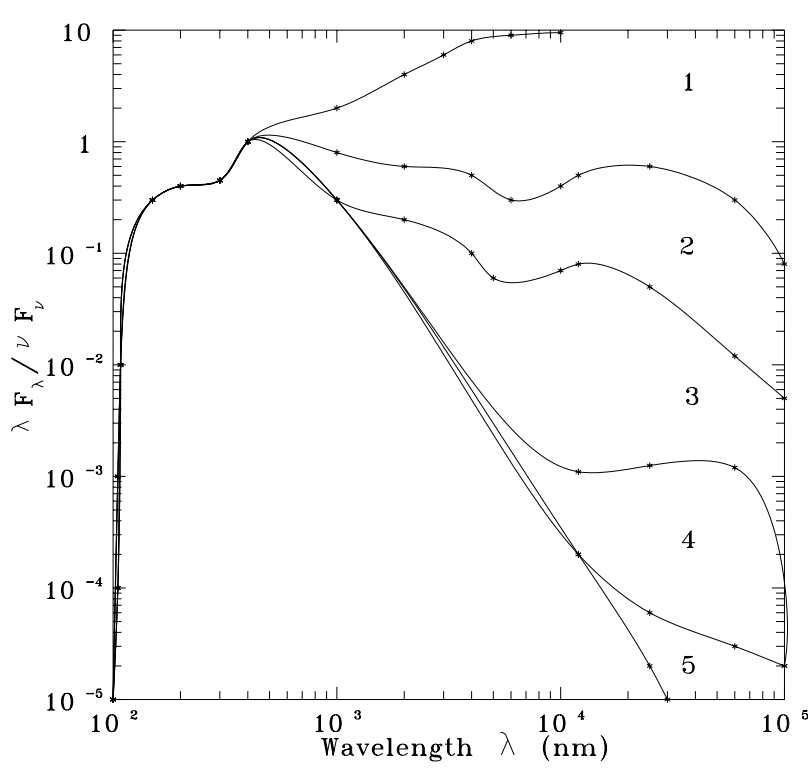

Fig. 19. Different types of SEDs for young stars

It is shown that for $85 \%$ of the sample stars there is a general relation between the degree of optical polarzation and the infrared colour index $(V-L)_{\text {obs }}$ and the colour excess $E(V-L)$ due to the contribution of a circumstellar dust shell. We show that on average HAEBE stars exhibit larger near IR excesses than TT stars due to the difference in the amount of hot dust in their CS shells. Clear differences are found also in the polarization distribution for the groups of TT and HAEBE stars that occur for the same reason. On the other hand, most of the young early-type stars which might be classified as Vega-type or $\beta$-Pic type or post HAEBE stars (i.e. comparable young stars near the end of the pre-MS evolutionary phase or young MS stars) as well as young MS solar-type stars show statistically smaller near IR excesses and polarization than HAEBE and TT stars. From the analysis of polarimetric and photometric behaviour of Vega-type stars we show that this group is at present inhomogeneous. Some objects might be considered as genuinely MS stars and they have small polarization and no IR excesses whereas others have a little higher level of polarization and some near IR excesses. It is felt that the latter objects are in the transition stage from HAEBE stars to young MS stars. The behaviour of HAEBE and TT stars with Algol-like minima of brightness indicates that most of them are positioned on the diagram in the region in which $85 \%$ of all young stars are located.

We show that none of Be stars, Mira Ceti stars, earlytype supergiants and MS stars lie within the relation derived for young objects but were distributed in separate groups on the diagram which reflects either absence of hot dust in their envelopes (if it exists) or differences in CS shell configuration.

All available data on the rotational velocities for young stars allow us to conclude that no correlation exists between $p_{\text {obs }}$ and $v \sin i$ for TT stars and for HAEBE stars. Slight changes in $v \sin i$ distributions for $\mathrm{TT} \Longleftrightarrow$ young solar-type stars are well explained in terms of the canonical theory of stellar evolution. For $v \sin i$ distributions in HAEBE and classical Be stars it is reasonably safe to suggest that these distributions diverge considerably. Classical Be stars are much more rapid rotators even in comparison with young Herbig Be and B Vega-type stars.

Analysis of polarimetric characteristics of early-type stars from our sample indicates that a few different groups of objects may be distinguished taking into account the value of polarization and polarimetric variability as well as the values of near IR excesses. We suppose that this differentiation is caused by the changes in structure and geometry of dust circumstellar shells around the stars and these changes are caused by evolution of the dust circumstellar environment.

We also found that most TT stars which are accompanied by "active" CS disks show significantly larger polarization and near IR excesses than other TT stars and possibly average polarization and IR excesses decrease with the disk's evolution. We suggest that the dissipation of CS matter with decreasing optical depth may be well explained in terms of a combination of infall and outflow.

As follows from the analysis there is a definite sequence of changes in polarization values from the group of young stars with flat or rising IR spectra through the group with decreasing IR spectra to the groups with small IR excesses and further to the group of stars which contains the objects which are close to or on the MS.

Finally we conclude that there is a clear evidence of changes in polarimetric behaviour of stars during the evolution from young stars to MS.

Acknowledgements. I'd like to express my sincere thanks to Dr. M.A. Pogodin (Pulkovo Observatory) for useful discussions and to Prof. A. Evans (Keele University) and Dr. G. Penny (Glasgow University) for the help in the improving of the text. The research described in this paper was made possible in part by the 99-02-16336 grant of RFBR. This research has made use of the VizieR Service and SIMBAD database, operated at Centre de Données Astronomiques de Strasbourg, France.

\section{References}

AAT, 1999, (www.sal.wisc.edu/WUPPE/polcats/aatobs.ra)

Abt H.A., Morrell N.I., 1995, ApJS 99, 135

Adams F.C., Lada C.J., Shu F.H., 1987, ApJ 312, 788

Ageorges N., Eckart A., Monin J.-L., Menard F., 1997, A\&A 326,632

van den Ancker M.E., de Winter D., Tjin A Djie H.R.E., 1998, A\&A 330, 145

Andriasyan R.R., Makarov A.H., 1989, Astrophysics 30, 101 
Artymowicz P., Burrows C., Paresce F., 1989, ApJ 337, 494

Axon D.J., Ellis R.S., 1976, MNRAS 177, 499

Barbier R., Swings J.P., 1982, Proc. IAU Symp. 98, Be stars, Jaschek M., Groth H.-G. (eds.). Dordrecht, p. 241

Bastien P., 1982, A\&AS 48, 153

Bastien P., 1985, A\&AS 59, 277

Bastien P., Menard F., Bertout C., 1996, JR Astron. Soc. Can. 90, 310

Bastien P., Robert C., Nadeau R., 1989, ApJ 339, 1089

Beckwith S.V.W., Sargent A.I., Chini R.S., Güsten R., 1990, AJ 99,924

Berdyugin A.V., 1993, Izv. KrAO 87, 107

Berdyugin A.V., Berdyugina S.V., Grinin V.P., Minikulov N.Kh., 1990, Sov. Astron. 67, 812

Bergner Yu.K., Miroshnichenko A.S., Yudin R.V., et al., 1986, Astron. Tsirc. 1459, 5

Bergner Yu.K., Miroshnichenko A.S., Krivtsov A.A., et al., 1993, Peremennye Zvezdy 23, 163

Bergner Yu.K., Miroshnichenko A.S., Yudin R.V., et al., 1995, A\&AS 112, 221

Bergner Yu.K., Miroshnichenko A.S., Yudin R.V., et al., 1990, Astrophysics 32, 109

Berrilli F., Corciulo G., Ingrosso G., et al., 1992, ApJ 398, 254

Bertout C., Harder S., Malbet F., Mennessier C., 1996, AJ 112, 2159

Beskrovnaya N.G., Pogodin M.A., Yudin R.V., et al., 1998, A\&AS 127, 243

Beskrovnaya N.G., Pogodin M.A., Miroshnichenko A.S., et al., 1999, A\&A 343, 163

Beskrovnaya N.G., Pogodin M.A., Najdenov I.D., Romanyuk I.I., 1995, A\&A 298, 585

Beskrovnaya N.G., Pogodin, 1999, A\&A (in preparation)

Bjorkman K.S., Schulte-Ladbeck R.E., 1994, ASP Conf. Ser. 62,74

Blondel P.F.C., Talavera A., Tjin A Djie H.R.E., 1993, A\&A 268, 624

Bouchert P., Swings J.P., 1981, in Proc. IAU Symp. 98, Be stars, Jaschek M., Groth H.-G. (eds.). Dordrecht, p. 241

Bouvier J., 1990, AJ 99, 946

Bouvier J., Appenzeller I., 1992, A\&AS 92, 481

Böhm T., Catala C., 1994, ASP Conf. Ser. 62, 219

Böhm T., Catala C., 1995, A\&A 301, 155

Breger M., 1974, ApJ 188, 53

Breger M., 1976, ApJ 204, 789

Breger M., 1977, ApJ 215, 119

Breger M., Gehrz R.D., Hackwell J.A., 1981, ApJ 248, 963

Briot D., Robichon N., 1998, Ap\&SSL 233, 47

Brooke T.Y., Tokunaga A.T., Strom S.E., 1993, AJ 103, 656

Brown A.G.A., Verschueren W., 1997, A\&A 319, 811

Calvet N., Giampapa M.S., Barri G., et al., 1985, ApJ 293, 575

Carpenter K.G., Slettebak A., Sonneborn G., 1984, ApJ 286, 741

Casanova S., Montmerle T., Feigelson E.D., Andre Ph., 1995, ApJ 439, 752

Casey B.W., Mathieu R.D., Suntzeff N.B., 1993, AJ 105, 2276

Chavarria-K C., 1981, A\&A 101, 105

Chini R., 1981, A\&A 99, 346

Chini R., Krügel E., Shustov B., et al., 1991, A\&A 252, 220

Chini R., Krügel E., Kreysa E., 1990, A\&A 227, L5

Chevalier C., Ilovaisky S.A., 1998, A\&A 330, 201

Chrysostomou A., Menard F., Gladhill T.M., et al., 1997, MNRAS 285, 750
Clarke D., Smith R.A., Yudin R.V., 1998, A\&A 336, 604

Clarke D., Smith R.A., Yudin R.V., 1999, A\&A 345, 547

Cohen M., Kuhi L., 1979, ApJS 41, 743

Cohen M., Schvartz R., 1976, MNRAS 174, 137

Cohen M., Fuller G.A., 1985, ApJ 296, 620

Cohen M., Emerson J.P., Beichman C.A., 1989, ApJ 339, 455

Corporon P., Lagrange A.-M., 1999, A\&AS 136, 429

Coté J., Waters L.B.F.M., 1987, A\&A 176, 93

Coulson I.M., Walther D.M., Dent W.R.F., 1998, MNRAS 296, 934

Daniel J.-Y., 1980a, A\&A 87, 204

Daniel J.-Y., 1980b, A\&A 86, 198

Daniel J.-Y., 1982, A\&A 111, 58

Davies J.K., Evans A., Boode M.F., Whittet D.C.B., 1990, MNRAS 247, 517

Davis R., Strom K.M., Strom S.E., 1983, AJ 88, 1644

Drissen L., Bastien P., St.-Louis N., 1989, AJ 97, 814

Drew J.E., Busfield G., Hoare M.G., et al., 1997, MNRAS 286 , 538

Dolginov A.Z., Gnedin Yu.N., Silant'ev N.A., 1995, in: Propagation and polarization of radiation in Cosmic Media. Gordon and Breach Publishers

Donati J.-F., Semel M., Carter B.D., Rees D.E., Cameron A.C., 1997, MNRAS 291, 658

Dougherty S.M., Taylor A.R., Clark T.A., 1991, AJ 102, 1753

Dudorov A.E., Pudritz R.E., 1994, ASP Conf. Ser. 62, 381

Dunkin S.K., Barlow M.J., Ryan S.G., 1997, MNRAS 286, 604

Dyck H.M., Forrest W.J., Gillet F.G., et al., 1971, ApJ 165, 57

Dzhakusheva K.G., Rspaev F.K., 1991, Astron. Tsirk. 1551, 11

Eiroa C., Garson F., Miranda L.F., 2000, in Proc. Euroconference on "Disks, Planetesimals and Planets", Eiroa C., de Winter D. and Mahoney J. (eds). ASP Conf. Ser. (in press)

Hipparcos and Tycho Catalogues, ESA SP-1200

Fajardo-Acosta S.B., Telesco C.M., Knacke R.F., 1998, AJ 115, 2101

Finkenzeller U., 1985, A\&A 151, 340

Franchini M., Covino E., Stalio R., et al., 1992, A\&A 256, 525

Friedemann C., Gürtler J., Reimann H.-G., 1995, A\&A 300, 269

Fuente A., Martin-Pintado J., Bachiller R., Neri R., Palla F., 1998, A\&A 334, 253

Gahm G.F., Liseau R., Gullbring E., Hartstein D., 1993, A\&A 279,477

Gahm G.F., Loden K., Gullbring E., Hartstein D., 1995, A\&A 301, 89

Gameiro J.F., Lago M.T.V.T., 1993, MNRAS 265, 359

Garrison L.M., Anderson C.M., 1978, ApJ 221, 601

Gauvin L.S., Strom K.M., 1992, ApJ 385, 217

Gezari D.Y., Pitts P.S., Schmitz M., 1999, CIO5 (vizier.ustrasbg.fr/cgi-bin/vizier?-source=II/225/catalog)

Giampapa N., Calvet N., Imhoff C.L., et al., 1981, ApJ 251, 113

Ghosh K., Iyengar K.W.K., Ramsey B.D., Austin R.A., 1999, AJ 118, 1061

Gladhill T.M., Scarrott S.M., 1989, MNRAS 236, 139

Gnedin Yu.N., Kiselev N.N., Pogodin M.A., et al., 1992, SvA Lett. 18, 182

Grady C.A., Perez M.R., Talavera A., et al., 1996, A\&AS 120, 157

Grasdallen G., Joyce R., Knacke R.F., et al., 1975, AJ 80, 117 
Gray R.O., Corbally C.J., 1998, AJ 116, 2530

Grillo F., Sciortiono S., Micela G., et al., 1992, ApJS 81, 795

Grinin V., 1998, in Proc. of the ESO Workshop "Cyclical Variability in Stellar Winds" held in Garching, Kapel L., Fulerton A.W. (eds.). Springer, p. 164

Grinin V., Nata A., Tambovtseva L., 1996, A\&A 313, 857

Grinin V.P., Kiselev N.N., Minikulov N.H., Chernova G.P., 1988, Sov. Astron. 14, 283

Grinin V.P., Kiselev N.N., Minikulov N.H., Chernova G.P., Voshchinnikov N.V., 1991, Ap\&SS 186, 283

Grinin V.P., 1994, ASP Conf. Ser. 62, 63

Grinin V.P., Kiselev N.N., Minikulov N.Kh., 1989, Pis'ma Astron. Zh. 15, 1028

Grinin V.P., Kolotilov E.A., Rostopchina A.N., 1995, A\&AS 112,457

Grinin V.P., Kozlova O.V., 2000, Astrophysics (submitted)

Grinin V.P., 2000, in Proc. Euroconference on "Disks, Planetesimals and Planets", Eiroa C., de Winter D. and Mahoney J., ASP Conf. Ser. (in press)

Gullbring E., Barwing H., Chen P.S., Gahm G.F., Bao M.X., 1996, A\&A 307, 791

Gullbring E., Gahm G.F., 1996, A\&A 308, 8211

Halbedel E.M., 1996, PASP 108, 833

Hall J.S., Mikesell A.H., 1953, Publ. US Naval Obs., XVII, Pt.I, 1

Hamann F., Person, S.E., 1992, ApJ 394, 628

Heiles C., 2000, AJ (accepted)

Herbig G.H., Bell K.R., 1988, Lick Obs. Bull. 1111, 1

Herbig G.H., 1960, ApJS 4, 337

Herbig G.H., 1977, ApJ 214, 747

Herbig G.H., Jones B.F., 1983, AJ 88, 1040

Hillenbrand L.A., Strom S.E., Vrba F.J., Keene J., 1992, ApJ 397,613

Howarth I.D., Siebert K.W., Hussain G.A.J., Prinja R.K., 1997, MNRAS 284, 265

HPOL, 1999, (www.sal.wisc.edu/HPOL/pbotgttype.html)

Huovelin J., Linnaluoto S., Piirola V., Tuominen I., Virtanen H., 1985, A\&A 152, 357

Huovelin J., Saar S.H., Tuominen I., 1988, ApJ 329, 882

Hutchinson M.G., Albinson J.S., Barrett P., et al., 1994, A\&A 285,883

Ivezic Z., Miroshnichenko A.S., Elitzur M., 1998, Ap\&SSL 233, 227

Jaschek C., 1998, Ap\&SSL 233, 61

Jensen E.L.N., Mathieu R.D., 1997, AJ 114, 301

Jian S.K., Bhatt H.C., 1995, A\&AS 111, 399

Kardapolov V.I., Rspaev F.K., Nosov I.V., 1988, SvA 32, 630

Kardapolov V.I., Rspaev F.K., 1990, Astron. Tsirk. 1544, 15

Kardapolov V.I., Pavlova L.A., Rspaev F.K., 1991, SvA 35, 278

Kardapolov V.I., Rspaev F.K., 1991, Astron. Tsirk. 1547, 13

Kenyon S.J., Hartmann W., 1991, Harw.-Smith. Center for Astroph., Prepr. No. 3276

Kerschbaum F., 1995, A\&AS 113, 441

Kilkenny D., Whittet D.C.B., Davies J.K., et al., 1985, SAAO Cirk. 9, 55

Kiselev N.N., Minikulov N.Kh., Chernova G.P., 1991, Astrofizika 34, 181

Knapic A., Bergeat J., 1997, A\&A 321, 236

Kopatskaya E.N., Shulov O.S., 1978, Tr. Astron. Obs. of Leningrad Univ. 34, 94
Krasnobabtsev V., 1981, Izv. KrAO 43, 93

Lamers H.J.G.L.M., Zickgraf F.-J., de Winter D., Houziaux L., Zorec J., 1998, A\&A 340, 117

Lecavelier des Etanges A., Vidal-Madjar A., Burki G., 1997, A\&A 328, 311

Lecavelier des Etanges A., Ferlet R., Vidal-Madjar A., 1997, A\&A 328, 602

Leinert C., Richichi A., Haas M., 1997, A\&A 318, 472

Leroy J.L., 1993, A\&AS 101, 551

Lucas T.W., Roche P.F., 1998, MNRAS 299, 699

Magnier E.A., Waters L.B.F.M., Groot P.J., et al., 1999, A\&A 346,441

Malfait K., Bogaert E., Waelkens C., 1998, A\&A 331, 211

Mannings V., 1994, MNRAS 271, 587

Mannings V., Sargent A.I., 1997, ApJ 490, 792

Mannings V., Barlow M.J., 1998, ApJ 497, 330

Marchenko S.V., Moffat A.F.J., van ded Hucht K., et al., 1998, A\&A 331, 1022

Mathewson D.S., Ford V.F., Klare G., et al., 1978, Bull. Iform. CDS 14, 115

Matsumuta M., Seki M., Kawabata K., 1999, AJ 117, 429

McLean I.S., Brown J.C., 1978, A\&A 69, 291

Mekkaden M.V., 1998, A\&A 340, 135

Menard F., Bastien P., 1992, AJ 103, 564

Men'shchikov A.B., Henning Th., 1997, A\&A 318, 879

Men'shchikov A.B., Henning T., Fischer O., 1999, ApJ 519, 257

Merin B., Montesinos B., 2000, in Proc. Euroconference on "Disks, Planetesimals and Planets", Eiroa C., de Winter D. and Mahoney J. (eds.), ASP Conf. Ser. (in press)

Miroshnichenko A.S., Yudin R.V., Yakubov S.D., 1992, Astron. Astroph. Trans. 1, 211

Miroshnichenko A.S., 1998, Ap\&SSL 233, 145

Miroshnichenko A.S., Fremat Y., Houziaux L., 1998, A\&AS 131,469

Miroshnichenko A.S., 1996, A\&A 312, 941

Miroshnichenko A.S., Mulliss Ch.L., Bjorkman K.S., et al., 1999a, MNRAS 302, 612

Miroshnichenko A.S., Gray R.O., Vieira S.L.A., et al., 1999b, A\&A 347, 137

Mitskevich A.S., 1995, A\&A 298, 231

Mirzoyan L.V., 1982, Astrofizika 18, 463

Miyake K., Nakagama Y., 1995, ApJ 441, 361

Moneti A., Pipher J.L., Helfer H.L., et al., 1984, ApJ 282, 508

Monin J.-L., Menard F., Duchkne G., 1998, A\&A 339, 113

Moujtahid A., Zorec J., Hubert A.M., 1998, Ap\&SSL 233, 57

Mundt R., 1980, A\&AS 39, 245

Mundt R., 1984, ApJ 280, 749

Myers, et al., 1998, ApJ 492, 703

Nata A., Grinin V.P., Mannings V., Ungerechts H., 1997, ApJ 491, 885

Nata A., Grinin V.P., Mannings V., 1999, in "Protostars and Planets IV"

Neri L.J., Chavarria-K C., de Lara E., 1993, A\&AS 102, 201

Oudmaijer R.D., van der Veen W.E.C.J., Waters L.B.F.M., et al., 1992, A\&AS 96, 625

Oudmaijer R.D., Drew J.E., 1999, MNRAS 305, 1660

Penston H.V., 1973, ApJ 183, 505

Petrov P.P., Zaijtseva G.V., Efimov Yu.S., et al., 1999, A\&A 341,553

Petrova N.N., Shevchenko V.S., 1987, Sov. Astron. Lett. 13, 686 
Piirola V., Scaltriti F., Coyne G.V. S.J., 1994, ASP Conf. Ser. 62,78

Pirzkal N., Spillar E.J., Dyck H.M., 1997, ApJ 481, 392

Plavec M., 1976, In Proc. of IAU Symp. 70, Slettebak A. (ed.), p. 439

Poeckert R., Bastien P., Landstreet J.D., 1979, AJ 84, 812

Pogodin M., 1998, in Proc. of the ESO Workshop "Cyclical Variability in Stellar Winds" held in Garching, Kapel L., Fulerton A.W. (eds.). Springer, p. 143

Ray T.P., Sargent A.I., Beckwith S.V.W., et al., 1995, ApJ 440, L89

Rostopchina A.N., 1999, AZh 76, 136

Rostopchina A.N., Grinin V.P., Okazaki A., et al., 1997, A\&A 327,145

Rostopchina A.N., Grinin V.P., Shakhovskoy D.N, 2000, in Proc. Euroconference on "Disks, Planetesimals and Planets", Eiroa C., de Winter D. and Mahoney J. (eds.). ASP Conf. Ser. (in press)

Rucinski S.M., Krautter J., 1983, A\&A 121, 217

Rydgren A.E., Vrba F.J., 1981, AJ 86, 1069

Rydgren A.E., Vrba F.J., 1983, AJ 88, 1017

Rydgren A.E., Strom S.E., Strom K.M., 1976, ApJS 30, 307

Rydgren A.E., Schmeltz J.T., Vrba F.J., 1982, ApJ 256, 168

Schulte-Ladbeck R., 1983, A\&A 120, 203

Serkowski K., 1969, ApJ 158, L107

Serkowski K., Mathewson D.S., Ford V.L., 1975, ApJ 196, 261

Shevchenko V.S., 1989, Herbig Ae/Be stars, FAN, Tashkent

Silvester R.J., Skinner C.J., Barlow M.J., Mannings V., 1996, MNRAS 279, 915

SIMBAD database, 1999 (simbad.u-strasbg.fr/SIMBAD)

Simon T., Herbig G., Boesgaard A.M., 1985, ApJ 293, 555

Sitko M.L., Grady C.A., Lynch D.K., Russel R.W., Hanner M.S., 1999, ApJ 510, 408

Skrutskie M.F., Meyer R., Whalen D., Hamilton C., 1996, AJ 112,2168

Slettebak A., Collins G.W. II., Boyce P.B., et al., 1975, ApJS 29,137

Slettebak A., 1982, ApJS 50, 55

Smith M.D., 1994, A\&A 287, 523

Soderblom D.R., Stauffer J.R., MacGregor K.B., Jones B.F., 1993, ApJ 409, 624

Solano E., Montesinos B., Mora A., 2000, in Proc. Euroconference on "Disks, Planetesimals and Planets", Eiroa C., de Winter D. and Mahoney J. (eds.). ASP Conf. Ser. (in press)

Stecklum B., Henning T., Feldt M., et al., 1998, AJ 115, 767

Strom K.M., Strom S.E., Edwards S., et al., 1989, AJ 97, 1451

Sun Yi. Li., Jaschek M., Andrillat Y., Jaschek C., 1985, A\&AS 62,309
Swings J.P., 1981, A\&A 98, 112

Tamura M., Sato S., 1989, AJ 98, 1368

Thé P.S., de Winter D., Perez M.R., 1994, A\&AS 104, 315

Thé P.S., Perez M.R., Voschinnikov N.V., van den Ancker M.E., 1996, A\&A 314, 233

Uesugi A., Fukuda I., 1982, Revised Catalogue of Stellar Rotational Velocities, Kyoto

Vieira S.L.A., Pogodin M.A., Franco G.A.P., 1999, A\&A 345, 559

Voshchinnikov N.V., Marchenko P.E., 1982, Sov. Astron. 59, 1115

Voshchinnikov N.V., Grinin V.P., 1988, Astrofizika 34, 181

Voshchinnikov N.V., Karjukin V.V., 1994, A\&A 288, 883

Vrba F.J., Schmidt G.D., Hintzen P.M., 1979, ApJ 227, 185

Vrba F.J., Strom S.E., Strom K.M., 1976, AJ 81, 958

Vrba F.J., Coyne G.V., Tapia S., 1993, AJ 105, 1010

Waters L.B.F.M., Marlborough J.M., 1992, A\&A 256, 195

Winkler H., 1997, MNRAS 287, 481

de Winter D., van den Ancker M.E., 1997, A\&AS 121, 275

de Winter D., Perez M.R., 1998, Ap\&SSL 233, 269

Whittet D.C.B., Assendorp R., Prusti T., et al., 1991, A\&A 251,521

Whittet D.C.B., Martin P.G., Hough J.H., et al., 1992, ApJ 386,562

Whittet D.C.B., Longmore A.J., McFadzean A.D., 1985, MNRAS 216, 45p

Whittet D.C.B., Gerakines P.A., Carkner A.L., et al., 1994, MNRAS 268, 1

Wolf B., Stahler O., 1985, A\&A 148, 412

Wolff S.C., Edwards S., Preston G.W., 1982, ApJ 252, 322

Wolff S.C., Simon T., 1997, PASP 109, 759

WUPPOL, 1999 (www.sal.wisc.edu/WUPPE/polcats/wuppol.html) Yudin R.V., 1988, Sov. Astr. 32, 652

Yudin R.V., 1990, in proc. "The infrared spectral region of stars", Jaschek C. and Andrillat Y. (eds.). Cambridge Univ. Press, p. 400

Yudin R.V., Evans A., 1998, A\&AS 131, 402

Yudin R.V., 1992, Ph.D. Thesis, Pulkovo Observatory

Yudin R.V., Clarke D., Smith R.A., 1999, A\&A 345, 547

Yudin R.V., Miroshnichenko A.S., 1988, Astrophysics 28, 91

Yudin R.V., 1996, A\&A 312, 234

Yudin R.V., 2000, A\&AS (submitted)

Zellner B., 1970, AJ 75, 182

Zickgraf F.-J., Schulte-Ladbeck R., 1989, A\&A 214, 274

Zinnecker H., Preibisch Th., 1994, ASP Conf. Ser. 62, 281

Zinnecker H., Preibisch Th., 1994, A\&A 292, 152

Zorec J., 1998, Ap\&SSL 233, 27

Zorec J., Briot D., 1997, A\&A 318, 443

Zuckerman B., Becklin E.E., 1993, ApJ 414, 793 